\title{
Analogue Wavelet Transform Based the Solution of the Parabolic Equation
}

\author{
Jean-Bosco Mugiraneza \\ Department of Computer Science, Faculty of Science and Technology, Kigali Independent University, ULK, P. O. Box \\ 2280 Kigali, Rwanda \\ mjbosco8@hotmail.com
}

Amritasu Sinha

Department of Mathematics, ETB, McMaster University, Canada

sinhaam@univmail.cis.mc master.ca

\begin{abstract}
In this paper we have proved that the solution of parabolic equation and its Fast Fourier Transform generate continuous wavelet transforms. Indeed, we have solved the parabolic equation using PDETool, exported its solution and coefficients to Matlab workspace. We have then imported the solution from workspace to signal processing tool. We have sampled the imported solution with the sampling frequency of $8192 \mathrm{~Hz}$ and applied the band pass filter with that frequency. The convolution of the sampled PDE solution with the impulse response of the band pass filter has generated wavelet transform. This algorithm computes the wavelet transform either directly of via Faster Fourier Transform. The computation of the FFT of the PDE solution has produced complex wavelet.
\end{abstract}

Index Terms - Wavelet Transform, Morlet Wavelet, PDE, FFT, Power Spectral Density, Matlab, Parabolic Equation.

\section{Introduction}

Wavelet analysis is an exciting new method for solving difficult problems in mathematics, physics and engineering. They find applications in wave propagation, data compression, signal processing, image processing, smoothing and image denoising, finger print verification, pattern recognition, computer graphics and multifractal analysis, the detection of aircraft and submarines. The wavelet transform is often compared with the Fourier Transform [1]. Fourier transform is a powerful tool for analyzing the components of a stationary signal (a stationary signal is where there is no change in the properties of signal). The Fourier transform is less useful in analyzing nonstationary signal (a non-stationary signal is a signal where there is change in the properties of signal). Wavelet transforms allow the components of a nonstationary signal to be analyzed. Wavelets also allow filters to be constructed for stationary and nonstationary signals [2], [3]. One of the main advantages of wavelets is that they offer a simu ltaneous localization in time and frequency domain whereas the standard Fourier transform is only localized in Frequency domain. Wavelets have the great advantage of being able to separate the fine details in a signal. Very small wavelets can be used to isolate very fine details in a signal, while very large wavelets can identify coarse details [1].

In general the any order derivative of the Gaussian function can be employed as a mother wavelet. On the other hand since the Gaussian function is a low frequency signal, it is not suitable to be a mother wavelet, and the mother is usually a high frequency signal [4]. The hyperbolic secant/hyperbolic tangent differential operator family can be useful when we need to process highly non-band limited signals, but where we do not need all of the power of the wavelets and/or where we wish to retain the ability to process functions locally (using a fundamentally local approximation). Such operators can be used:

- To calculate rough, but wide-ranging, frequency spectrum values of unknown signals.

- To represent high frequency signals approximately using only (relatively) slow integrations to correct the integration coefficients [5].

One of the fundamental questions of applied harmonic analysis is to obtain density conditions on the sequences required to discretize a continuous integral transform in a way that the resulting discretization is a frame in a certain Hilbert space. This question was sharply solved for the frames in the Berg mann-Fock [6], [7], [8] and in the Bergamann space [9]. However, in the case of the Wavelet and Gabor transforms, very little is known and only a few very special windows and analyzing wavelets are understood [10]. The short time Fourier (Gabor) transform with respect to a Gaussian window can be written in terms of the Bargmann transform, mapping iso metrically the space $L^{2}(R)$ onto 
the Barg mann -Fock space of the entire functions.This is the reason why everything is known about the geometry of sequences that generate frames by sampling the Gabor transform with Gaussian windows $g(t)=e^{-\pi t^{2}}$. A part from this example, the only cases where a description is known of the lattice sequences that generate frames are the hyperbolic secant $g(t)=(\cosh a t)^{-1}[11]$ and the characteristics function of an interval[12], which turned out to be the nontrivial problem. There is also a necessary condition for Gabor frames due to Ramanathan and Steger [13].

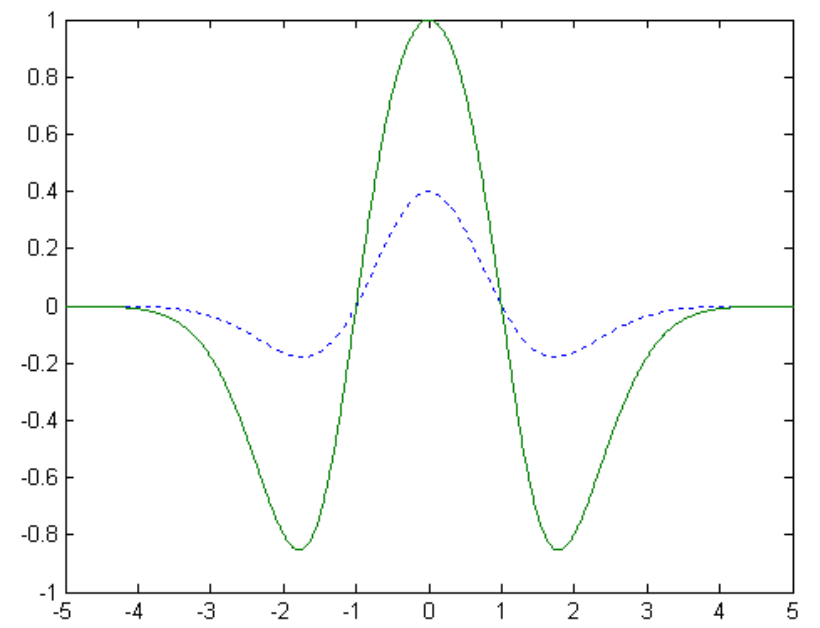

Fig. 1: $y_{1}(t)=\frac{1}{\sqrt{2 \pi}}\left(1-t^{2}\right) e^{-\frac{1}{2} t^{2}} \quad$ dotted curve; $y_{2}(t)=\left(1-t^{2}\right)\left(\cosh \left(-\frac{1}{2} t^{2}\right)\right)^{-1}$

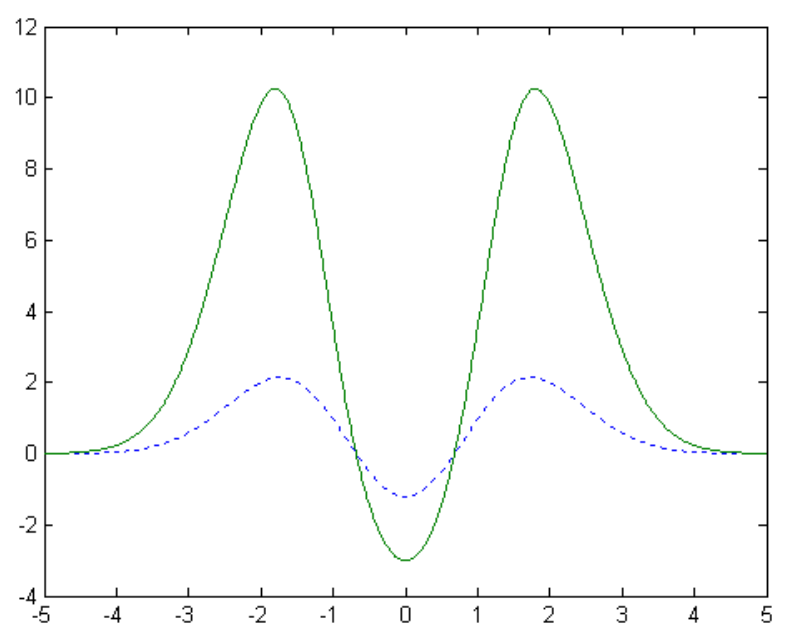

Fig. 2: $\sqrt{2 \pi}\left(t-16 t^{2}-3\right)$ dotted curve; $y_{2}(t)=\left(t^{4}+6 t^{2}-3\right)\left(\cosh \left(-\frac{1}{2} t^{2}\right)\right)^{-1}$
One of the reasons wavelets have found so many uses and applications is that they are especially attractive from the computational point of view. Computational efficiency of wavelets lies in the fact that wavelet coefficients in wavelet expansions for functions in $V_{0}$ (resolution subspace in $L^{2}\left(R^{d}\right)$ may be computed using matrix iteration, rather than by a direct computation of inner products: the latter would involve integration over $R^{d}$, and hence be computationally inefficient, if feasible at all. The deeper reason for why we can compute wavelet coefficients using matrix iteration is an important connection to the subband filtering method from signal/image processing involving digital filters, down-sampling and upsampling. In this setting filters may be realized as functions $m_{0}$ on a d-torus, e.g., quadrature mirror filters [14].

The wavelet transform of a signal $g(t)$ is defined as

$$
w(a, b)=\int_{-\infty}^{+\infty} g(t) \psi^{*}\left(\frac{t-b}{a}\right) \frac{d t}{a}
$$

(the asterisk means complex conjugation)

$$
\psi(\xi)=\frac{e^{\frac{\omega_{0}}{2}}}{\sqrt{2 \pi}} e^{-i \omega_{0} \xi} e^{\frac{-\xi^{2}}{2}}
$$

and the magnitude norm is used

$$
\int_{-\infty}^{+\infty}\left|\psi\left(\frac{t-b}{a}\right)\right| \frac{d t}{a}=e^{\frac{\omega_{0}}{2}}
$$

Eqn.(1) is a derivative of the diffusively smooth initial function [15]

$$
w(a, b)=a^{n} \frac{\partial^{n}}{\partial b^{n}} \int_{-\infty}^{+\infty} g(t) \psi_{0}\left(\frac{t-b}{a}\right) \frac{d t}{a}
$$

The wavelet transform is also a solution of the partial differential equation [16]

$$
\left(a \frac{\partial^{2}}{\partial b^{2}}-\frac{\partial}{\partial a}-i \omega_{0} \frac{\partial}{\partial b}\right) w(a, b)=0
$$

The initial value is $w(0, b)=g(b)$

In [17] the wavelet -image is written as a sum of the real part and imaginary parts

$$
w(a, b)=u(a, b)+v(a, b)
$$

In this representation Eqn.(3) will be converted to the following system of partial differential equations: 


$$
\begin{aligned}
& \frac{\partial u}{\partial a}=a \frac{\partial^{2} u}{\partial b^{2}}+\omega_{0} \frac{\partial v}{\partial b} \\
& \frac{\partial v}{\partial a}=a \frac{\partial^{2} v}{\partial b^{2}}-\omega_{0} \frac{\partial u}{\partial b}
\end{aligned}
$$

The initial values for the real valued signals are

$$
u(0, b)=g(b), v(a, b)=0
$$

The complex Morlet wavelet is given by:

$$
w_{a, b}(t)=\left[\begin{array}{c}
\cos \omega_{0}\left(\frac{t-a}{b}\right) \\
+\sin \omega_{0}\left(\frac{t-a}{b}\right)
\end{array}\right] h_{a, b}(t)
$$

$$
h_{a, b}(t)=\frac{1}{\sqrt{a}} e^{-\frac{1}{2}\left(\frac{t-a}{b}\right)^{2}}
$$

and $a$ and $b$ represents the translation and scale of the windowed function $h_{a, b}(t)$, respectively. The term $\frac{1}{\sqrt{a}}$ in different scales [18]. Fig.3 shows respectively the real and imaginary parts represented by:

$$
r(t)=\frac{1}{\sqrt{2 \pi}} \cos (2 \pi t)\left[\left(1-t^{2}\right) e^{-\frac{1}{2} t^{2}}\right]
$$

and

$$
i(t)=\frac{1}{\sqrt{2 \pi}} \sin (2 \pi t)\left[\left(1-t^{2}\right) e^{-\frac{1}{2} t^{2}}\right]
$$

Where
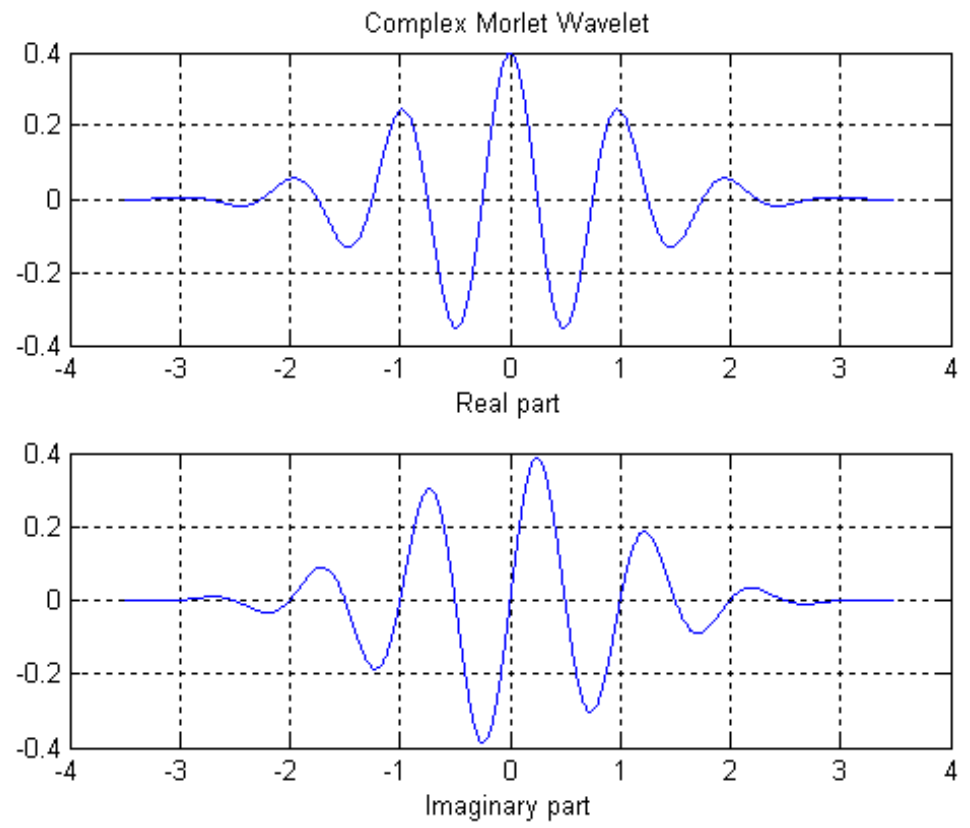

Fig. 3: Complex Morlet Wavelet

The present paper has the following sections: section two provides the solution of parabolic Equation with PDETool, section three deals with the analysis of the PDE solution using Fast Fourier Transform (FFT), and we will wind with the conclusion.

\section{Solving Parabolic Equation with PDETool}

Wavelets and their application for the solution of partial differential equations in physics were discussed in [19]. In this paper, we have solved the parabolic equation using PDETool. We have considered the parabolic equation $d \frac{\partial u}{\partial t}-\nabla(c \nabla u)+a u=f$

If $f=0$ then we say that the equation (9) is homogeneous.

In our study we have used the following PDE coefficients;

$$
a(t)=t^{3} ; c(t)=t ; d(t)=e_{;}^{-t} f(t)=\sin t
$$

where

$c(t)$ is the time varying coefficient of conductivity 
$d(t)$ is the time varying coefficient of diffusivity

The solution $u$ of the equation is represented in Fig.4 and Fig.5. We have then exported the time varying PDE coefficients $c(t), a(t), f(t)$ and $d(t)$ as well as the PDE solution to Matlab works pace.

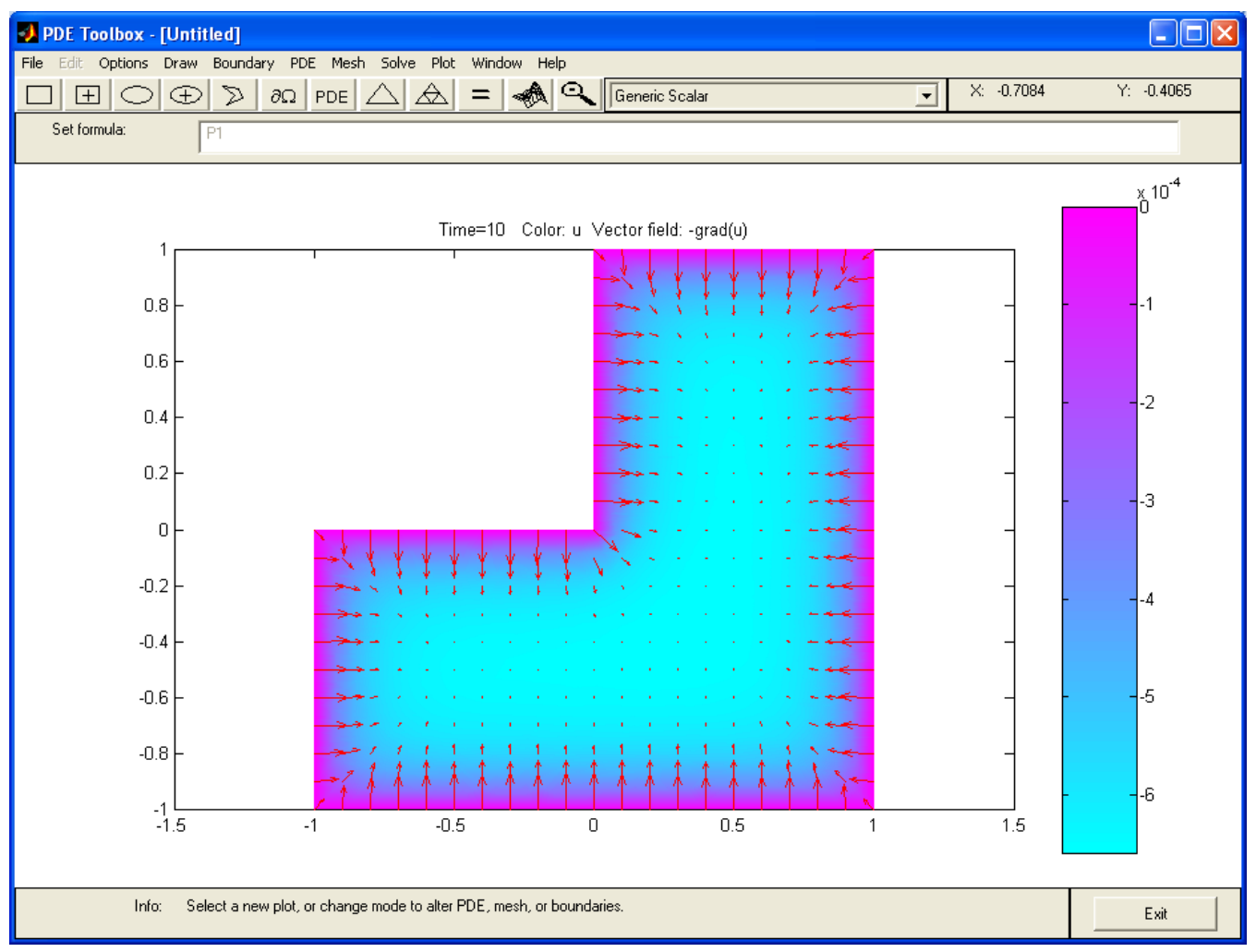

Fig. 4: PDE Solution with Arrow Plot

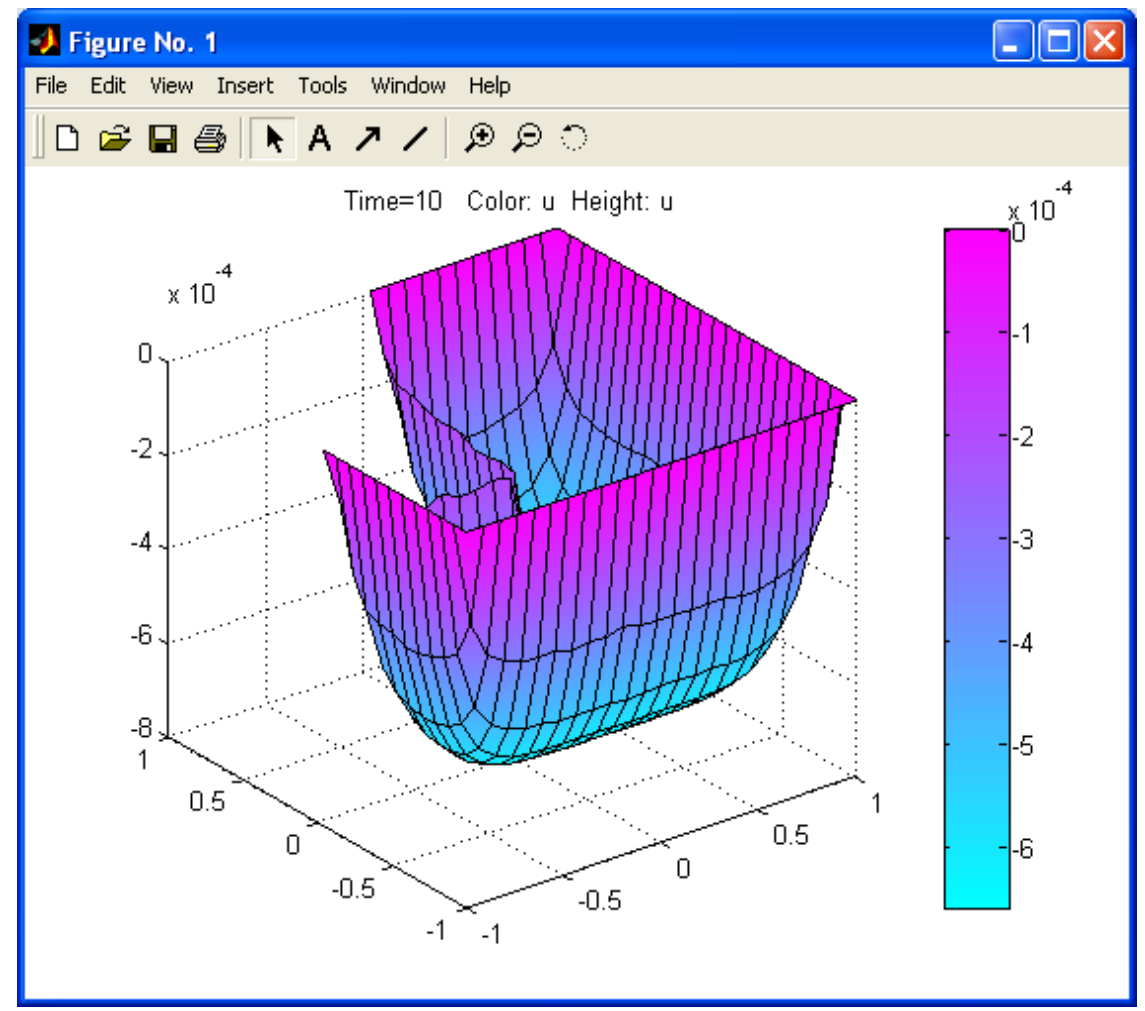

Fig. 5: PDE Solution with Height Plot 
The solution $u$ was imported from Matlab workspace to signal processing tool with the sampling frequency of $8192 \mathrm{~Hz}$. This tool has three main components: signal section view, filter section view and edit as well as spectra section. These are used to visualize waveforms and spectra of several signals and make a qualified filter design. Therefore, characteristic properties and desired parameters of the solution have been estimated. In order to use the PDE solution under these processes the solution was imported as a real array of consisting of 11 column index vectors each having 557 data points.

There are two ways from which one can generate analogue wavelet transform from PDE solution:

1. Either one can directly use the PDE solution and apply a properly designed band pass filter as per Fig.8. and Fig.9. or

2. Alternatively one can compute the Fast Fourier Transform of the PDE solution and apply the same filter.

$$
\text { Let } s(t)=\left[\begin{array}{lllll}
s_{1}(t) & s_{2}(t) & \cdot & \cdot & s_{n}(t)
\end{array}\right]
$$

be the sampling vector signal

$$
\text { Let } x(t)=\left[\begin{array}{llllll}
x_{1}(t) & x_{2}(t) & . & . & . & x_{n}(t)
\end{array}\right]
$$

be the sampled vector signal

$$
\text { Let } y(t)=\left[\begin{array}{lllll}
y_{1}(t) & y_{2}(t) & . & . & y_{n}(t)
\end{array}\right]
$$

be the response of the band pass filter

Let $f(t)$ be the impulse response of the band pass filter

$$
\text { Let } u=\left[\begin{array}{cccccc}
u_{11} & u_{12} & \cdot & \cdot & \cdot & u_{1 n} \\
u_{21} & u_{22} & \cdot & \cdot & \cdot & u_{2 n} \\
\cdot & \cdot & \cdot & \cdot & \cdot & \cdot \\
\cdot & \cdot & \cdot & \cdot & \cdot & \cdot \\
\cdot & \cdot & \cdot & \cdot & \cdot & \cdot \\
u_{m 1} & u_{m 2} & \cdot & \cdot & \cdot & u_{m n}
\end{array}\right]
$$

be $m \times n_{\mathrm{PDE}}$

solution matrix where $m=11_{\text {and }} n=557$

This data matrix is a real matrix, $u \in R^{m \times n}$, associated with $m \times n$ points of the PDE solution. In this section we attempt to express the sampled vector signal $x(t)$ in terms of sampling vector signal $s(t)$ as

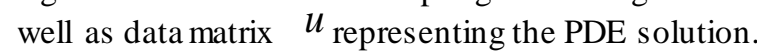

$$
\begin{aligned}
x_{1}(t) & =u_{11} s_{1}(t)+u_{21} s_{2}(t)+\ldots+u_{n 1} s_{n}(t) \\
& =\sum_{i=k=1}^{n} u_{i 1} s_{k}(t) \\
x_{2}(t) & =u_{12} s_{1}(t)+u_{22} s_{2}(t)+\ldots+u_{n 2} s_{n}(t) \\
& =\sum_{i=k=1}^{n} u_{i 2} x_{k}(t)
\end{aligned}
$$

$$
\begin{aligned}
x_{m}(t) & =u_{1 m} s_{1}(t)+u_{2 m} s_{2}(t)+\ldots+u_{n m} s_{n}(t) \\
= & \sum_{i=k=1}^{n} u_{i m} s_{k}(t)
\end{aligned}
$$

In vector form $x(t)=s(t) \times u$ can be written as:

$$
\left[\begin{array}{c}
x_{1}(t) \\
x_{2}(t) \\
\cdot \\
\cdot \\
x_{m}(t)
\end{array}\right]=\left[\begin{array}{cccccc}
u_{11} & u_{21} & \cdot & \cdot & \cdot & u_{m 1} \\
u_{12} & u_{22} & \cdot & \cdot & \cdot & u_{m 2} \\
\cdot & \cdot & \cdot & \cdot & \cdot & \cdot \\
\cdot & \cdot & \cdot & \cdot & \cdot & \cdot \\
\cdot & \cdot & \cdot & \cdot & \cdot & \cdot \\
u_{1 n} & u_{2 n} & \cdot & \cdot & \cdot & u_{m n}
\end{array}\right] \times\left[\begin{array}{c}
s_{1}(t) \\
s_{2}(t) \\
\cdot \\
\cdot \\
\cdot \\
s_{n}(t)
\end{array}\right]
$$

\section{Power of the sampled PDE solution}

From the definition of power the power associated to sampled PDE solution is given by:

$$
\begin{aligned}
& P_{x_{1}}=\left|x_{1}(t)\right|^{2}=\left|\sum_{i=k=1}^{n} u_{i 1} s_{k}(t)\right|^{2} \\
& P_{x_{2}}=\left|x_{2}(t)\right|^{2}=\left|\sum_{i=k=1}^{n} u_{i 2} s_{k}(t)\right|^{2}
\end{aligned}
$$

$P_{x_{m}}=\left|x_{m}(t)\right|^{2}=\left|\sum_{i=k=1}^{n} u_{i m} s_{k}(t)\right|^{2}$

Total power of the sampled PDE solution is: 


$$
\begin{aligned}
P_{x} & =\sqrt{\left(P_{x_{1}}\right)^{2}+\left(P_{x_{2}}\right)^{2}+\ldots .+\left(P_{x_{m}}\right)^{2}} \\
& =\sqrt{\sum_{j=1}^{m}\left(\left|x_{j}(t)\right|^{2}\right)^{2}} \\
& =\sqrt{\sum_{j=1}^{m}\left(\left|\sum_{i=k=1}^{n} u_{i j} s_{k}(t)\right|^{2}\right)^{2}}
\end{aligned}
$$

\section{Energy of the sampled PDE solution}

$$
\begin{aligned}
& E_{x_{1}}=\int_{-\infty}^{+\infty}\left|x_{1}(t)\right|^{2} d t=\int_{-\infty}^{+\infty}\left(\left|\sum_{i=k=1}^{n} u_{i 1} s_{k}(t)\right|^{2}\right) d t \\
& E_{x_{2}}=\int_{-\infty}^{+\infty}\left|x_{2}(t)\right| d t=\int_{-\infty}^{+\infty}\left|\sum_{i=k=1}^{n} u_{i 2} s_{k}(t)\right|^{2} d t \\
& \cdot \\
& E_{x_{m}}=\int_{-\infty}^{+\infty}\left|x_{m}(t)\right| d t=\int_{-\infty}^{+\infty}\left|\sum_{i=k=1}^{n} u_{i m} s_{k}(t)\right|^{2} d t
\end{aligned}
$$

Total energy of the sampled PDE solution is:

$$
E_{x}=\sqrt{\left(E_{x_{1}}\right)^{2}+\left(E_{x_{2}}\right)^{2}+\ldots .+\left(E_{x_{m}}\right)^{2}}
$$

From Eqn. (11) we obtain:

$$
\begin{aligned}
E_{x} & =\sqrt{\sum_{j=1}^{m}\left(\int_{-\infty}^{+\infty}\left|x_{1}(t)\right|^{2} d t\right)^{2}} \\
& =\sqrt{\sum_{j=1}^{m}\left(\int_{-\infty}^{+\infty}\left|\sum_{i=k=1}^{n} u_{i j} s_{k}(t)\right|^{2} d t\right)^{2}}
\end{aligned}
$$

By definition the response of the band pass filter is given by the convolution of the impulse response $f(t)$ of the band pass filter with the sampled vector

$$
\text { signal } x(t)=\left[\begin{array}{llllll}
x_{1}(t) & x_{2}(t) & . & . & x_{n}(t)
\end{array}\right] .
$$

Hence we get:

$$
y(t)=f(t) * x(t)=\int_{-\infty}^{+\infty} x(t) f(t-\tau) d \tau
$$

Apply the above definition to each column, we get:

$$
\begin{aligned}
y_{1}(t)=x_{1}(t) * f(t) & =\int_{-\infty}^{+\infty} x_{1}(t) f(t-\tau) d \tau \\
& =\int_{-\infty}^{+\infty}\left(\sum_{i=k=1}^{n} u_{i 1} s_{k}(t)\right) f(t-\tau) d \tau \\
& =\sum_{i=k=1}^{n} u_{i 1} \int_{-\infty}^{+\infty} s_{k}(t) f(t-\tau) d \tau
\end{aligned}
$$

$$
\begin{aligned}
y_{2}(t)=x_{2}(t) * f(t) & =\int_{-\infty}^{+\infty} x_{2}(t) f(t-\tau) d \tau \\
& =\int_{-\infty}^{+\infty}\left(\sum_{i=k=1}^{n} u_{i 2} s_{k}(t)\right) f(t-\tau) d \tau \\
& =\sum_{i=k=1}^{n} u_{i 2} \int_{-\infty}^{+\infty} s_{k}(t) f(t-\tau) d \tau \\
y_{m}(t)=x_{m}(t) * f(t) & =\int_{-\infty}^{+\infty} x_{m}(t) f(t-\tau) d \tau \\
& =\int_{-\infty}^{+\infty}\left(\sum_{i=k=1}^{n} u_{i m} s_{k}(t)\right) f(t-\tau) d \tau \\
& =\sum_{i=k=1}^{n} u_{i m} \int_{-\infty}^{+\infty} s_{k}(t) f(t-\tau) d \tau
\end{aligned}
$$

The filtered PDE solution $y(t)$ is shown in Fig. 10. The filtered PDE solution is a modulated signal. Hence we have generated analog continuous wavelet transform from PDE solution.

The Matlab signal processing tool shows that the modulated signal generated out of the PDE solution is a real signal. Its expression can be derived from the equation of complex mother Morlet equation given in Eqn. (7a) by equating the imaginary part to zero. Hence we get

$$
y_{i}(t)=w_{a_{i}, b_{i}}(t)=h_{a_{i}, b_{i}}(t) \cos \omega_{0}\left(\frac{t-a_{i}}{b_{i}}\right)
$$

We can therefore see that the filter effect has generated a wavelet transform that is proportional to the windowed function. 


\section{Power of the filtered sampled PDE solution}

$$
\begin{aligned}
& P_{y_{1}}=\left|y_{1}(t)\right|^{2}=\left|\sum_{i=k=1}^{n} u_{i 1} \int_{-\infty}^{+\infty} s_{k}(t) f(t-\tau) d \tau\right|^{2} \\
& P_{y_{2}}=\left|y_{2}(t)\right|^{2}=\left|\sum_{i=k=1}^{n} u_{i 2} \int_{-\infty}^{+\infty} s_{k}(t) f(t-\tau) d \tau\right|^{2} \\
& \text {. } \\
& P_{y_{m}}=\left|y_{m}(t)\right|^{2}=\left|\sum_{i=k=1}^{n} u_{i m} \int_{-\infty}^{+\infty} s_{k}(t) f(t-\tau) d \tau\right|^{2}
\end{aligned}
$$

Total Power of the filtered sampled PDE solution can be computed considering it as a vector with components.

$$
\begin{aligned}
P_{y} & =\sqrt{\left(P_{y_{1}}\right)^{2}+\left(P_{y_{2}}\right)^{2}+\ldots+\left(P_{y_{m}}\right)^{2}} \\
& =\sqrt{\sum_{j=1}^{m}\left(\left|y_{j}(t)\right|^{2}\right)^{2}} \\
P_{y} & =\sqrt{\sum_{j=1}^{m}\left(\left|\sum_{i=k=1}^{n} u_{i m} \int_{-\infty}^{+\infty} s_{k}(t) f(t-\tau) d \tau\right|^{2}\right)^{2}}
\end{aligned}
$$

\section{Energy of the filtered sampled PDE solution}

$$
\begin{aligned}
E_{y_{1}} & =\int_{-\infty}^{+\infty}\left|y_{1}(t)\right|^{2} d t \\
& =\int_{-\infty}^{+\infty}\left|\sum_{i=k=1}^{n} u_{i 1} \int_{-\infty}^{+\infty} x_{k}(t) f(t-\tau) d \tau\right|^{2} d t \\
E_{y_{2}} & =\int_{-\infty}^{+\infty}\left|y_{2}(t)\right|^{2} d t \\
& =\int_{-\infty}^{+\infty}\left|\sum_{i=k=1}^{n} u_{i 2} \int_{-\infty}^{+\infty} x_{k}(t) f(t-\tau) d \tau\right|^{2} d t
\end{aligned}
$$

$$
\begin{aligned}
E_{y_{m}} & =\int_{-\infty}^{+\infty}\left|y_{m}(t)\right|^{2} d t \\
& =\int_{-\infty}^{+\infty}\left|\sum_{i=k=1}^{n} u_{i m} \int_{-\infty}^{+\infty} x_{k}(t) f(t-\tau) d \tau\right|^{2} d t
\end{aligned}
$$

Total energy of the filtered sampled PDE solution can be computed considering that this energy is a vector. Then the magnitude of that energy is given by the following formula:

$$
E_{y}=\sqrt{\left(E_{y_{1}}\right)^{2}+\left(E_{y_{2}}\right)^{2}+\ldots+\left(E_{y_{m}}\right)^{2}}
$$

From Eqn. (14) we get:

$$
\begin{aligned}
E_{y} & =\sqrt{\sum_{j=1}^{m}\left(\int_{-\infty}^{+\infty}\left|y_{j}(t)\right|^{2} d t\right)^{2}} \\
& =\sqrt{\sum_{j=1}^{m}\left(\int_{-\infty}^{+\infty}\left|\sum_{i=k=1}^{n} u_{i 1} \int_{-\infty}^{+\infty} x_{k}(t) f(t-\tau) d \tau\right|^{2} d t\right)^{2}}
\end{aligned}
$$

The sampled PDE solution is shown in Fig.6. It was computed using music method of power computation. The power spectral density (PSD) of the PDE solution is shown in Fig. 7 with minimum frequency equal to half of the sampling frequency.

A stable band pass filter has been designed to analyze in this frequency range the PDE solution. Fig.8 indicates the pole-zero plot of the filter. Magnitude, phase response, step response, group phase delay and other properties of the same filter are shown in Fig.9.

The filtering process has eliminated noise successfully as indicated by power spectral densities (PSDs) of pre-filtering and post-filtering. The filtered signal is a modulated signal shown in Fig.10. The PSD of the filtered PDE solution is shown in Fig.11. 


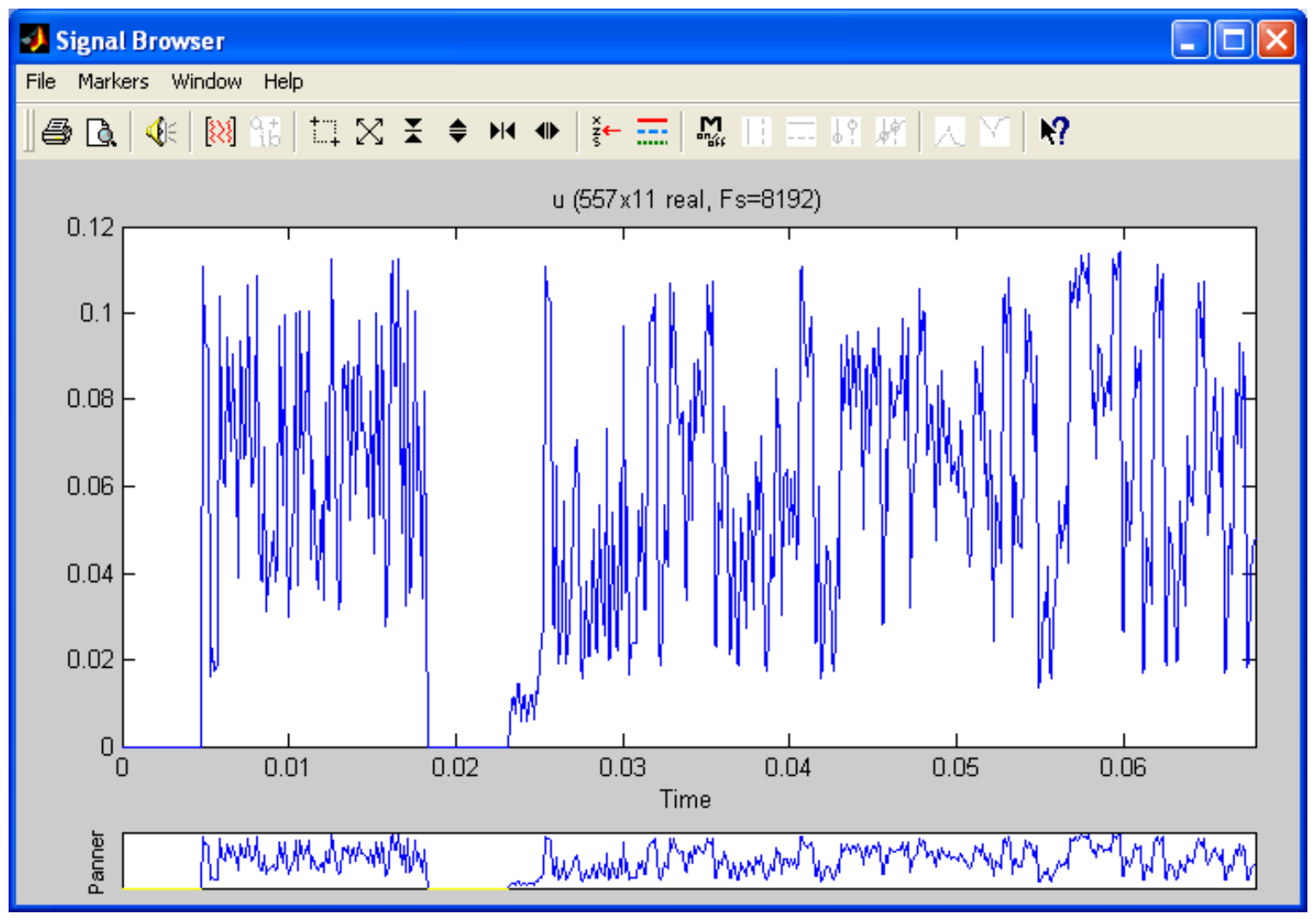

Fig. 6: Sampled PDE Solution for Column Index Vector 2

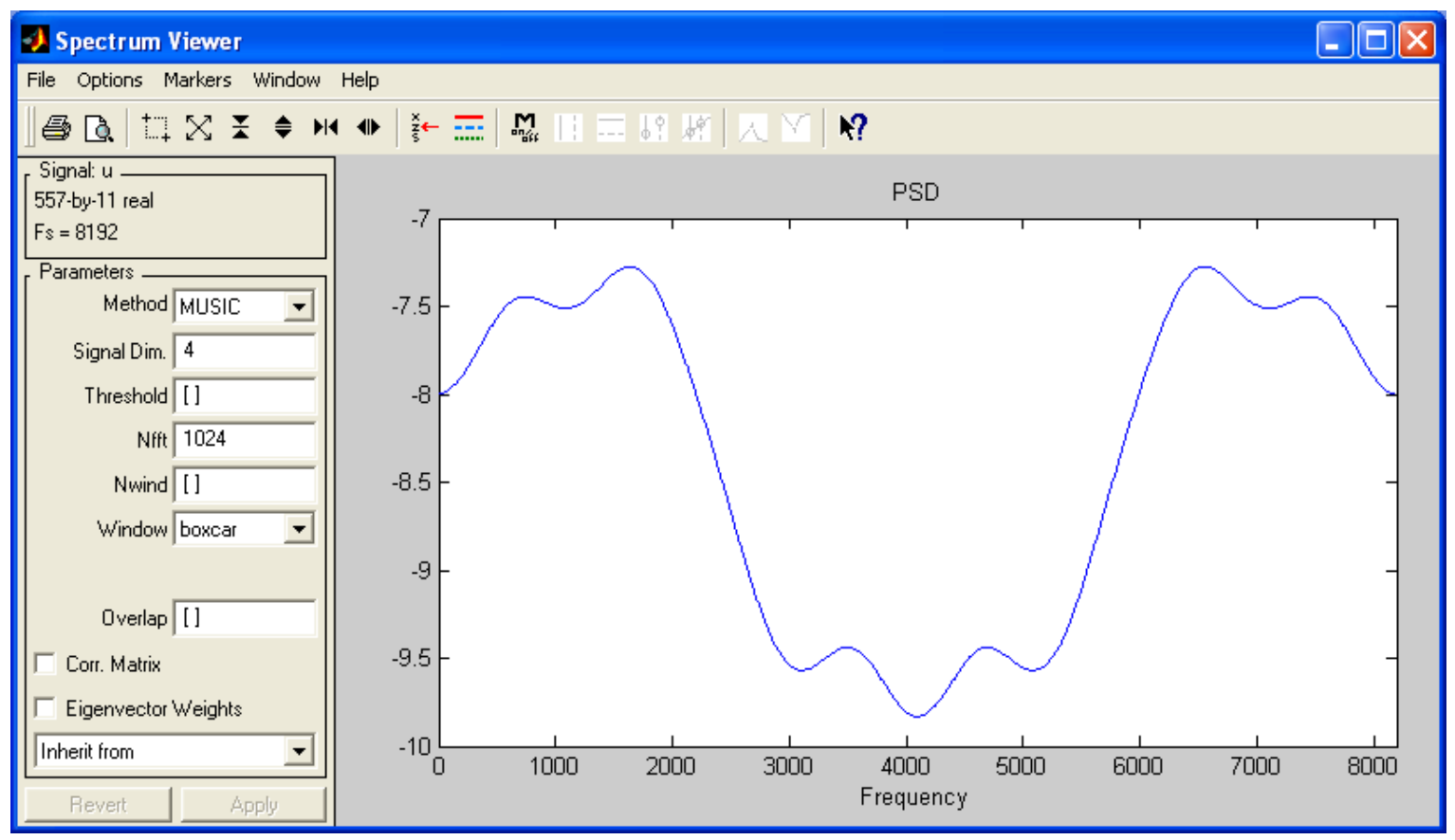

Fig. 7: PSD of Sampled PDE Solution 


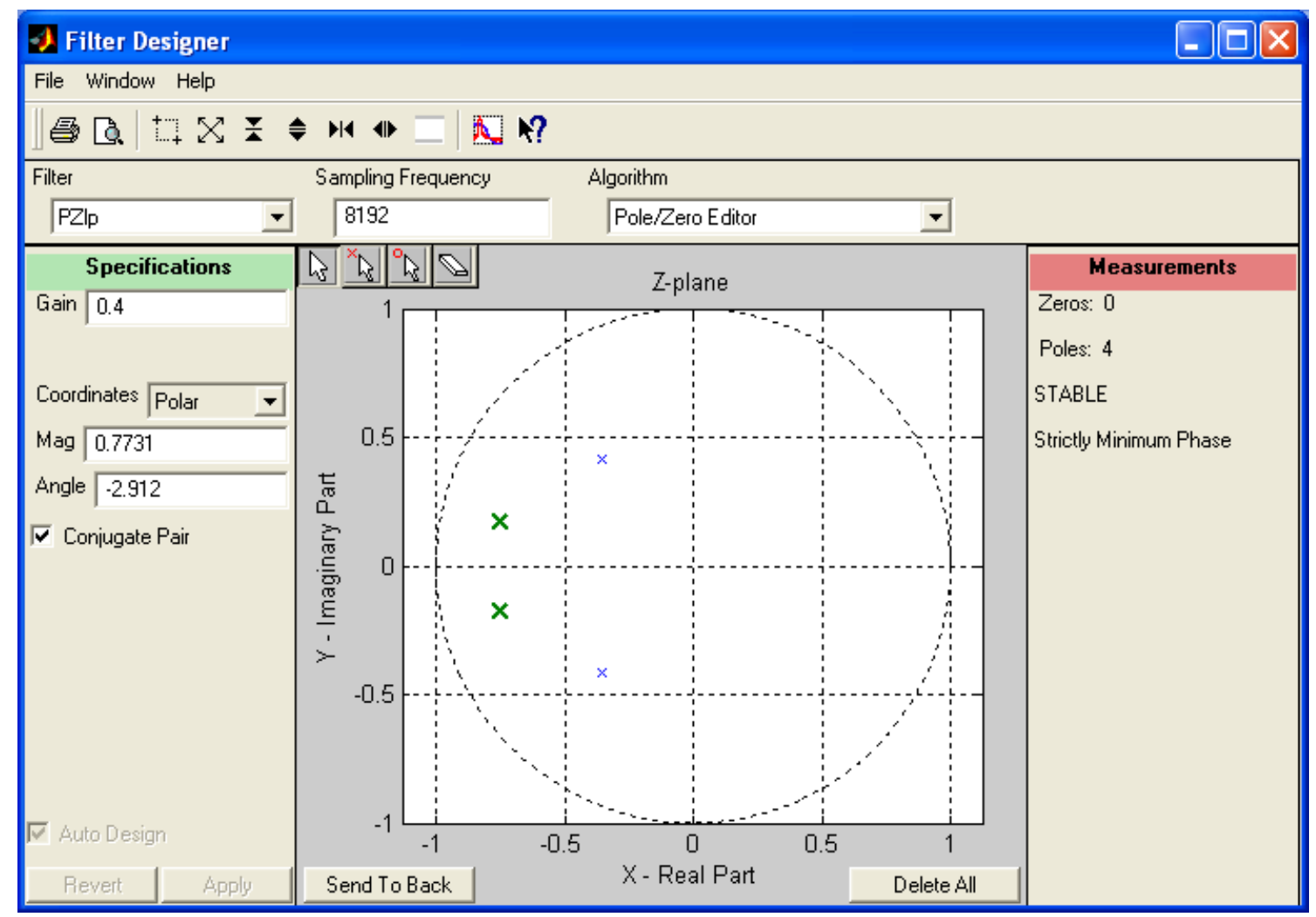

Fig. 8: Filter Pole-Zero Plot

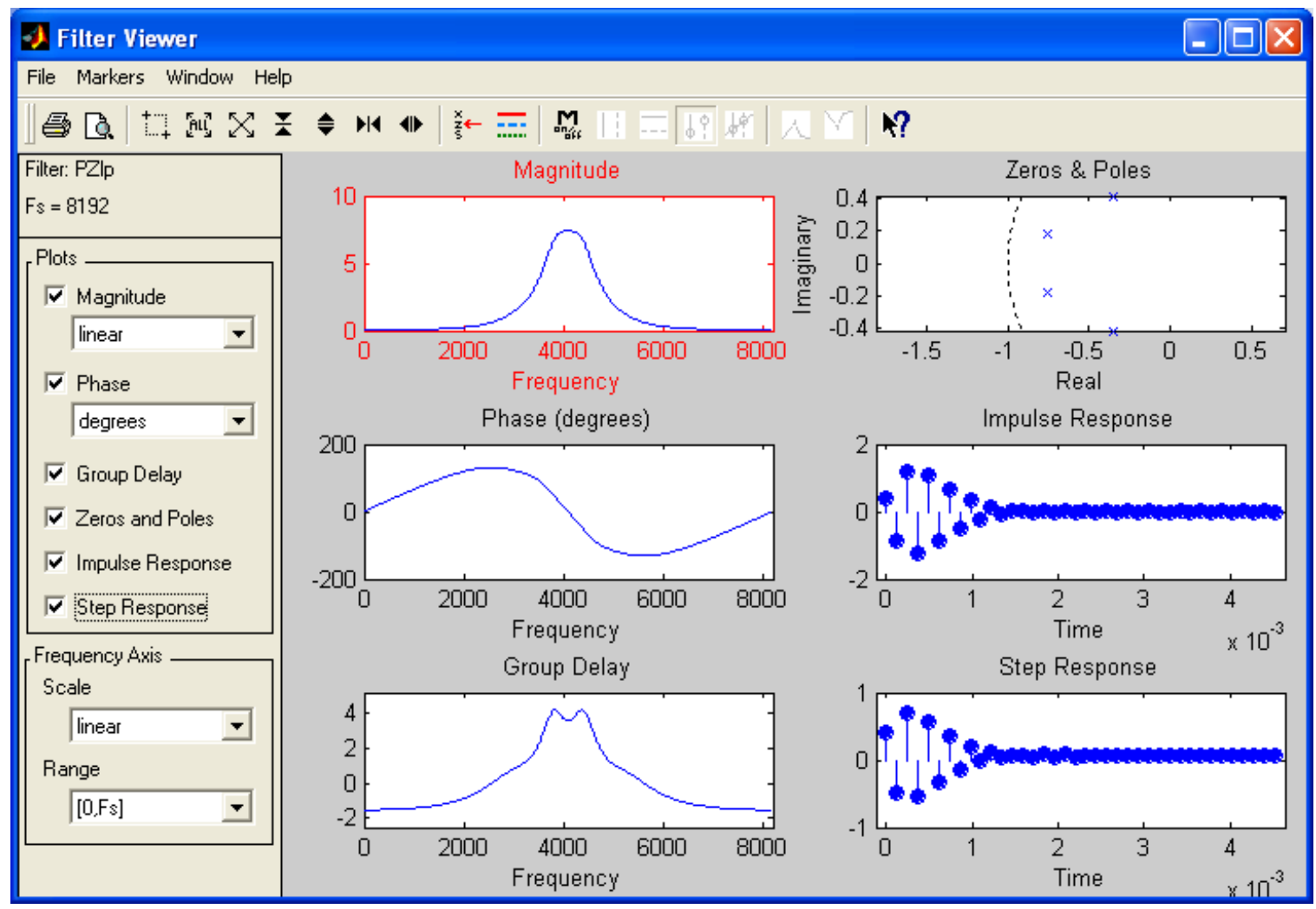

Fig. 9: Filter Characteristics 


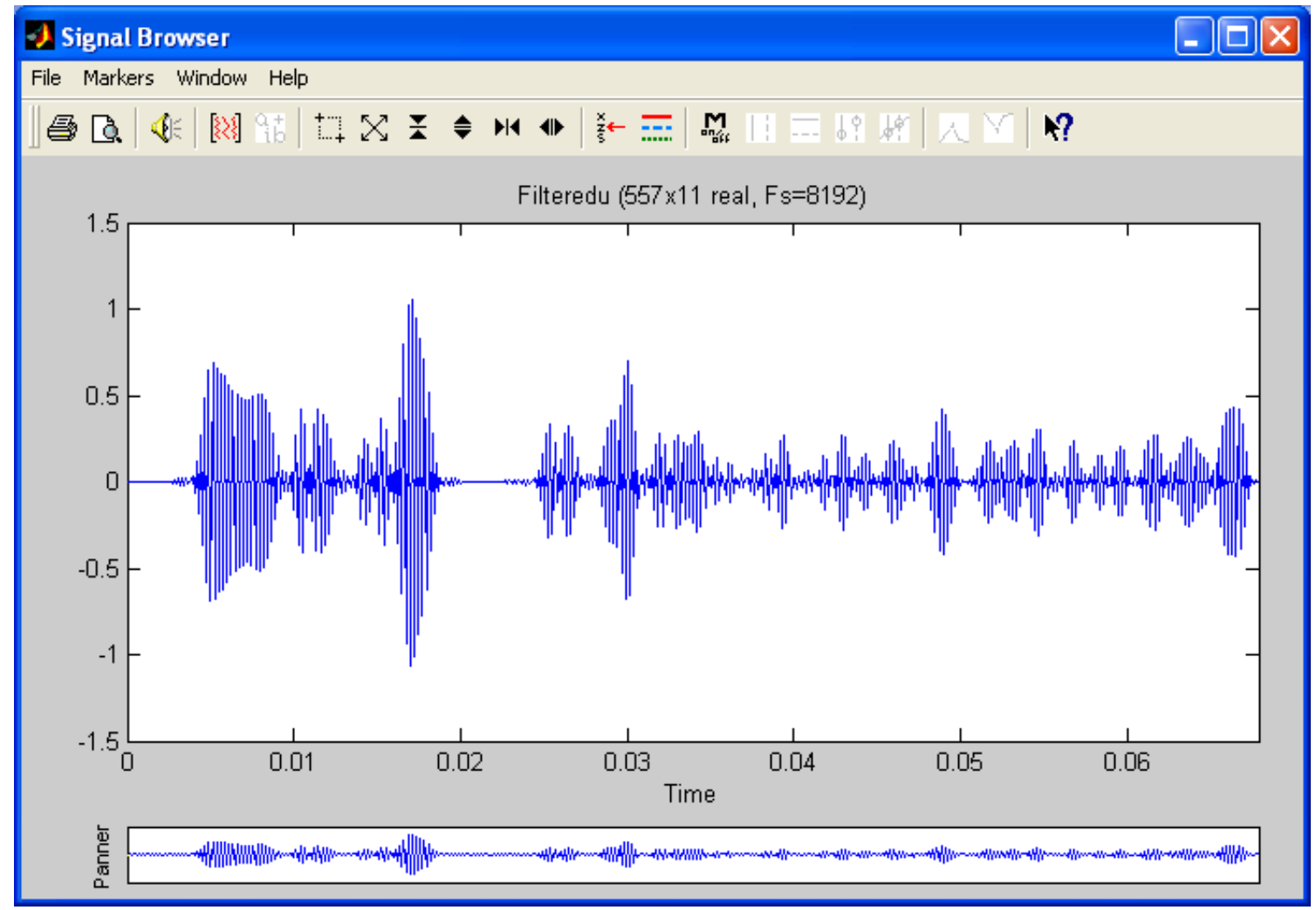

Fig. 10: Filtered PDE Solution for Column Index Vector 2

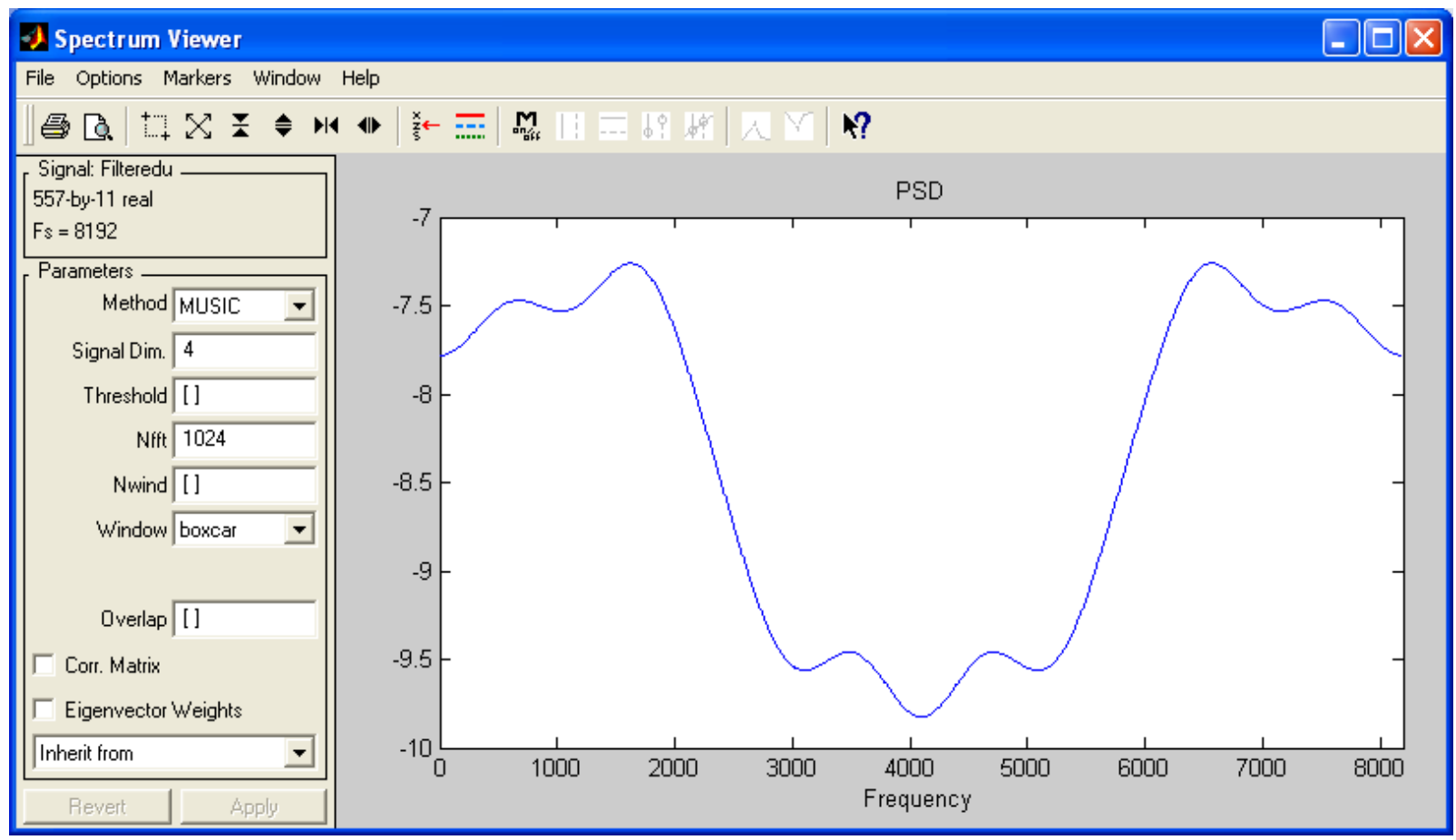

Fig. 11: PSD of Filtered PDE Solution

\section{Spectrum Analysis with the FFT}

Let us now explore the frequency analys is of the PDE solution through the computation of the Fast Fourier Transform. Unlike PDE solution discussed in the previous section the computation of FFT generates points which are complex in nature. The plot of $\mathrm{ftt}(\mathrm{u})$ is shown in Fig.12. The complex points of the FFT of the PDE solution are imported from Matlab workspace to Matlab signal processing tool with the same sampling 
frequency. Fig.13, Fig.14, Fig.15 and Fig.16 show the real the part, imaginary part, magnitude and phase angle of the Fast Fourier Transform of the PDE solution respectively.

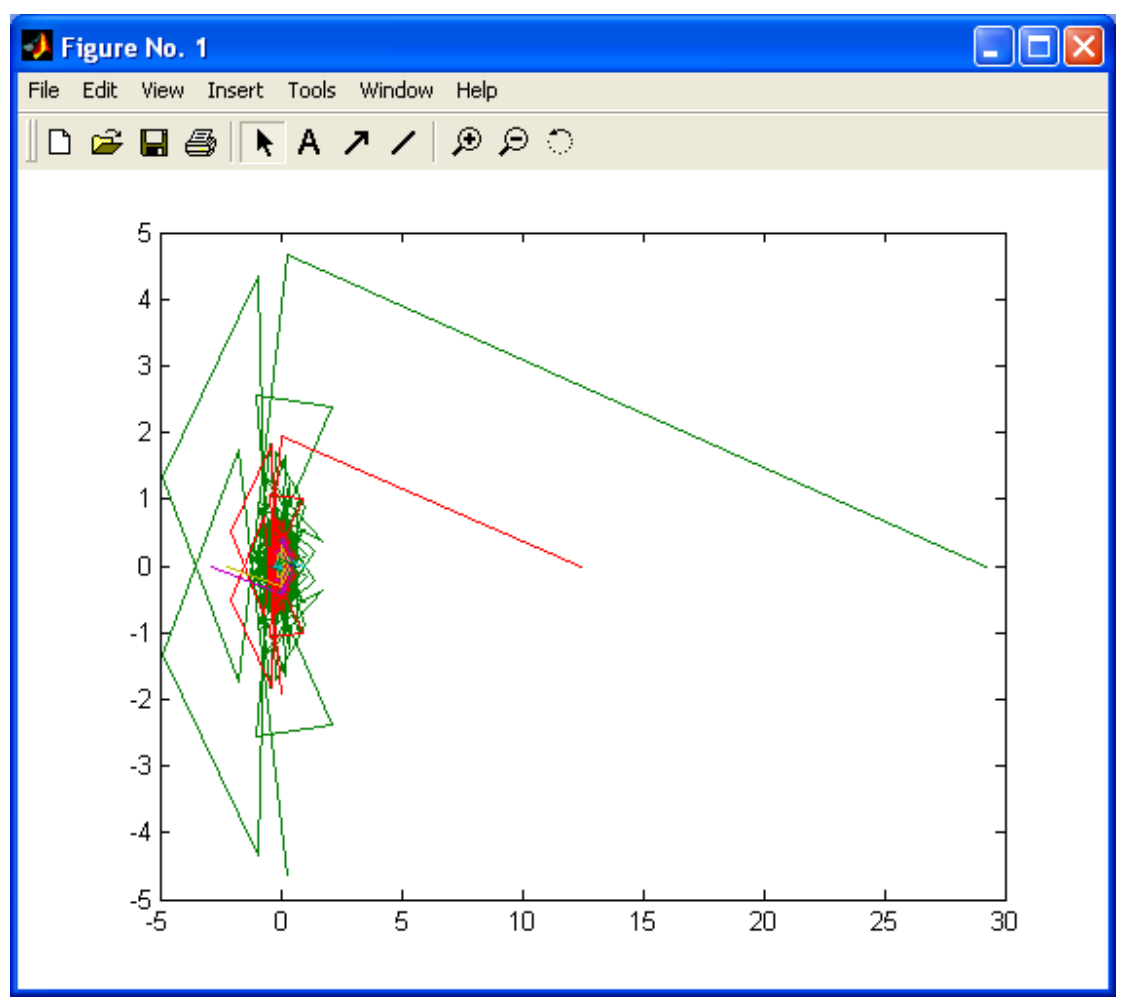

Fig. 12: FFT of PDE Solution

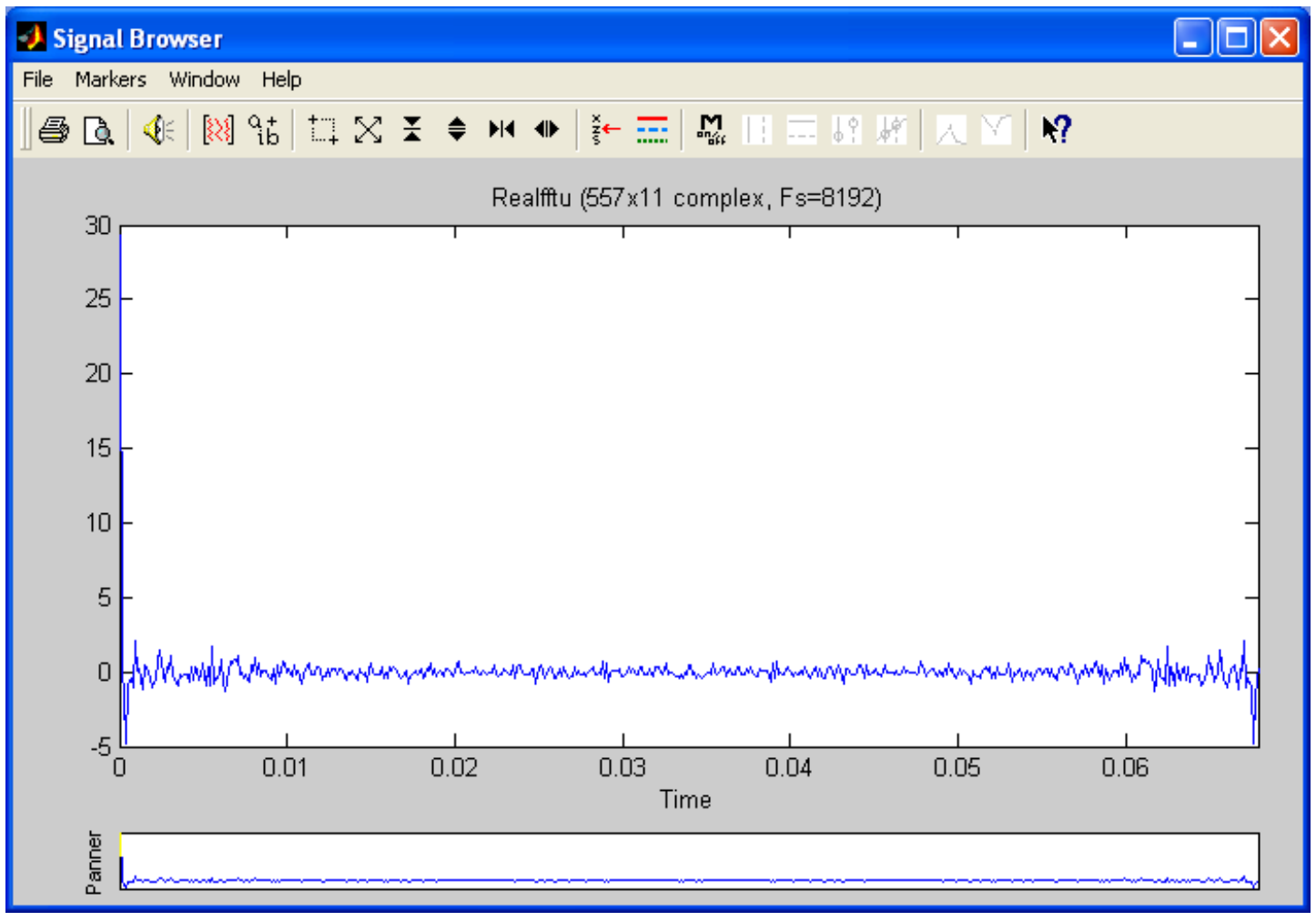

Fig. 13: Real Part of fft(u) for Column Index Vector 2 


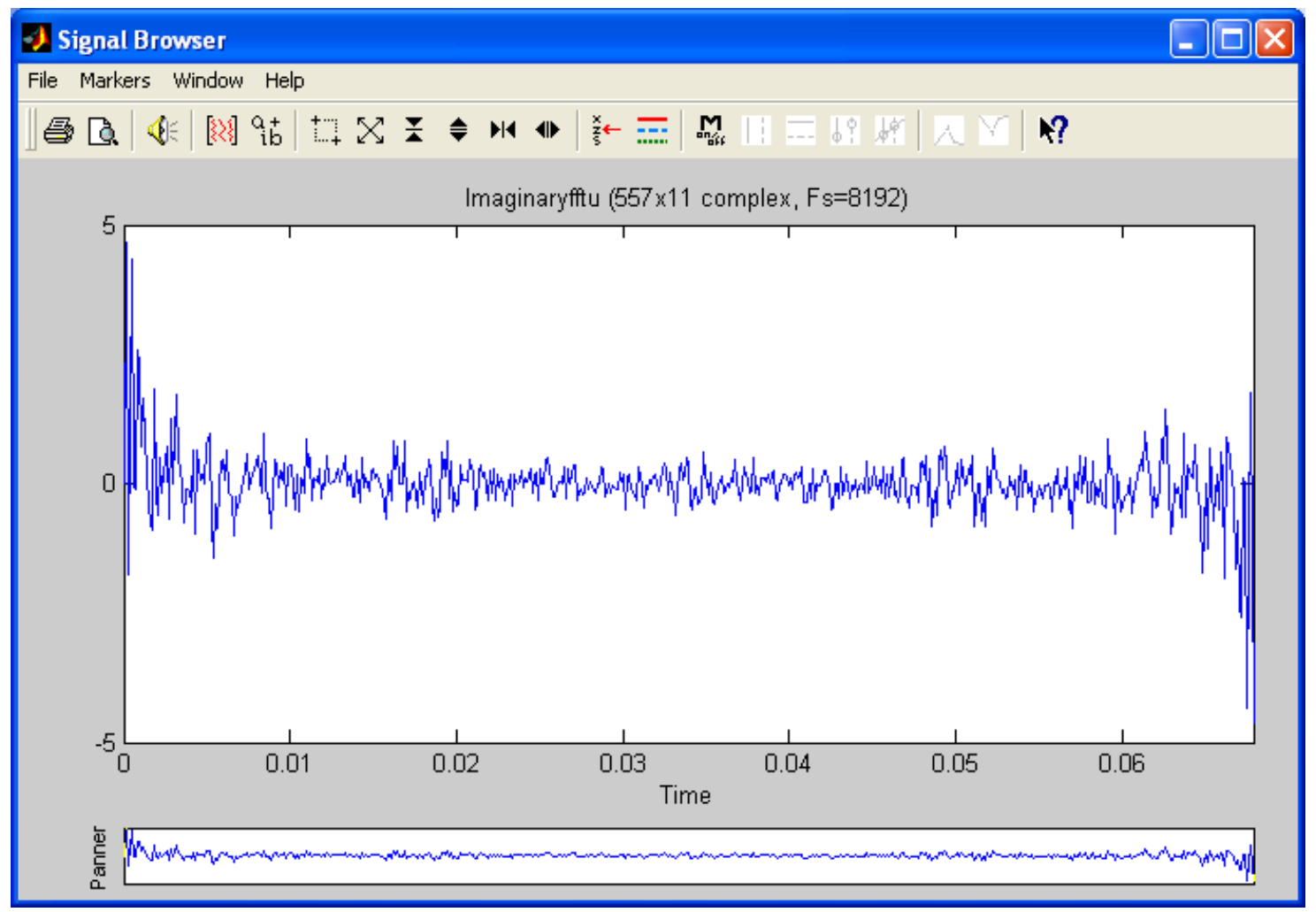

Fig. 14: Imaginary Part of fft(u) for Column Index Vector 2

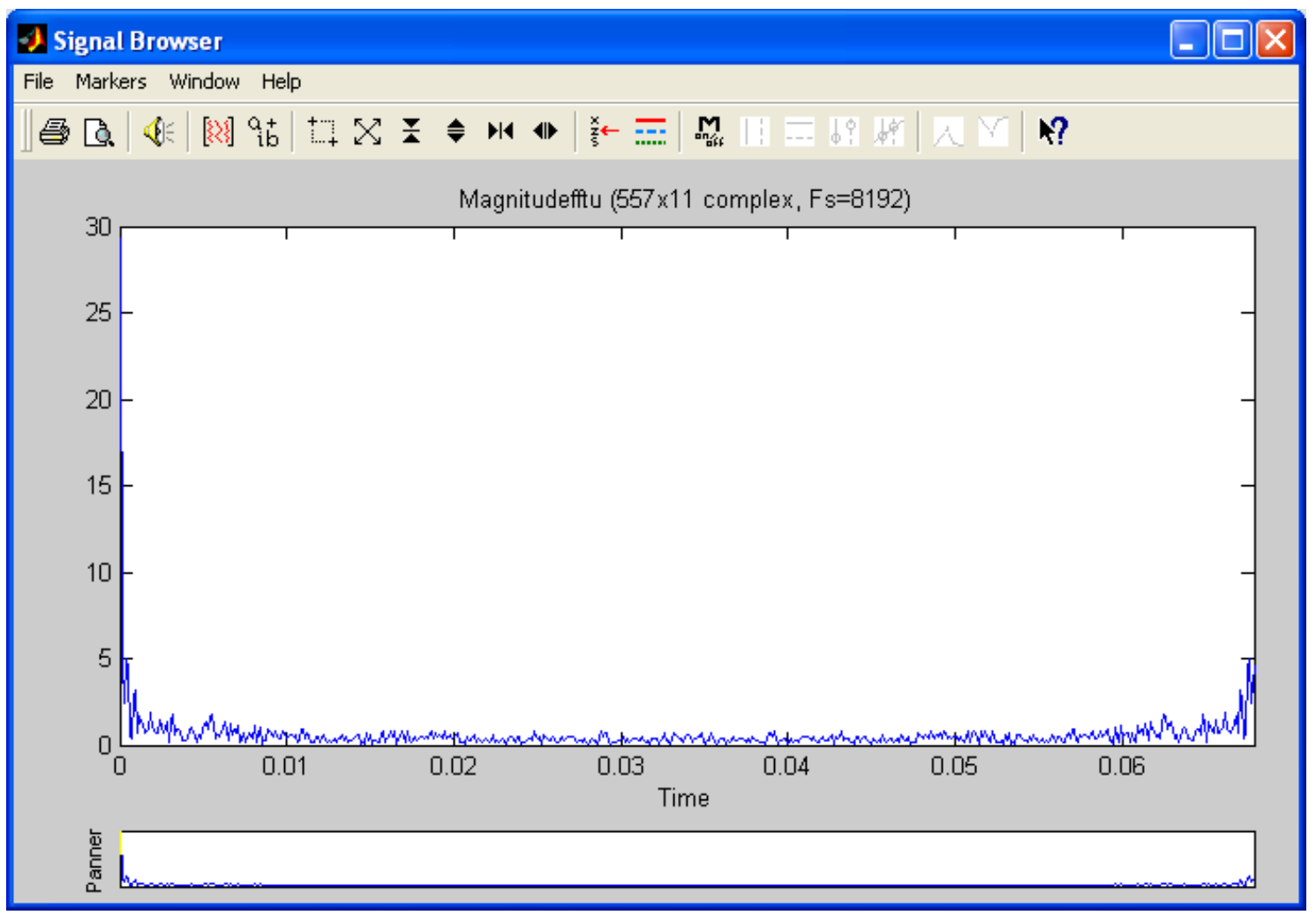

Fig. 15: Magnitude of $\mathrm{fft}(\mathrm{u})$ for Column Index Vector 2 


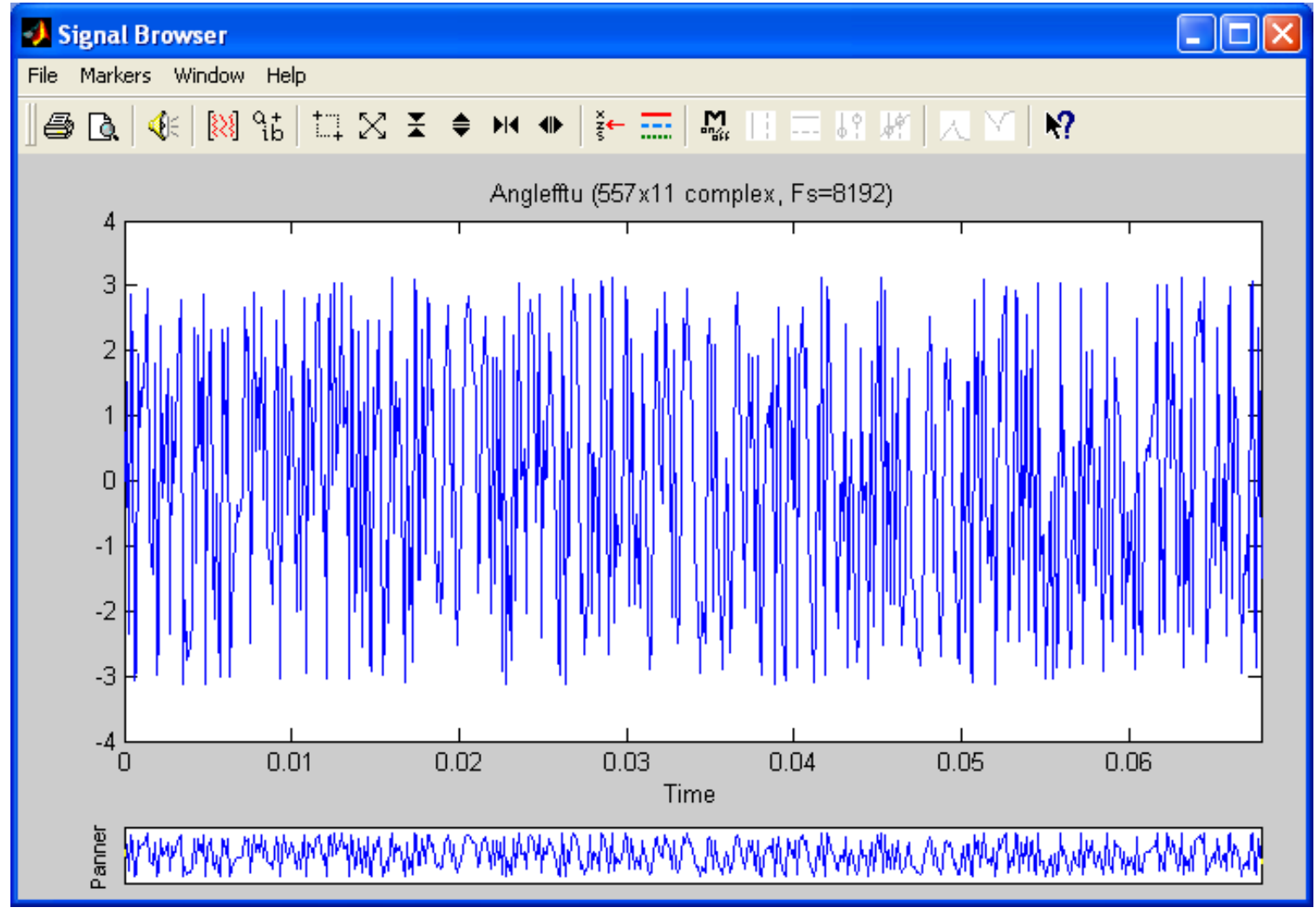

Fig. 16: Phase Angle of fft(u) for Column Index Vector 2

The FFT does not directly give you the spectrum of a signal. The FFT contains information between 0 and $f_{s}$, however, we know that the sampling frequency must be at least twice the highest frequency component. Therefore, the signal's spectrum should be entirely below $\frac{f_{s}}{2}$, the Nyquist frequency. Recall also that a real signal should have a transform magnitude that is symmetrical for positive and negative frequencies. So instead of having a spectrum that goes from 0 to $f_{s}$, it would be more appropriate to show the spectrum from $-\frac{f_{s}}{2}$ to $\frac{f_{s}}{2}$ [20].

The computation of the FFT allows the analysis of the PDE solution in frequency domain. It therefore provides additional information to the existing ones on the PDE solution, especially on the power spectral density. The filtered real part, imaginary part, magnitude and phase angle of the Faster Fourier Transform of the PDE solution are respectively shown in Fig.18, Fig.19, Fig.20 and Fig.21, whereas the power spectral density (PSD) of the filtered Fast Fourier Transform of the PDE solution is shown in Fig.22.

$$
\text { Let } s(t)=\left[\begin{array}{lllll}
s_{1}(t) & s_{2}(t) & . & . & s_{n}(t)
\end{array}\right] \text { be the }
$$
sampling vector signal
Let $c(t)=\left[\begin{array}{lllll}c_{1}(t) & c_{2}(t) & \cdot & \cdot & c_{n}(t)\end{array}\right]$ be the sampled vector signal

Let $w(t)=\left[\begin{array}{lllll}w_{1}(t) & w_{2}(t) & \cdot & \cdot & w_{n}(t)\end{array}\right]$ be the response of the band pass filter

Let $f(t)$ be the impulse response of the band pass filter

Let $f f t(u)=\left[\begin{array}{cccccc}z_{11} & z_{12} & \cdot & \cdot & \cdot & z_{1 n} \\ z_{21} & z_{22} & \cdot & \cdot & \cdot & z_{2 n} \\ \cdot & \cdot & \cdot & \cdot & \cdot & \cdot \\ \cdot & \cdot & \cdot & \cdot & \cdot & \cdot \\ \cdot & \cdot & \cdot & \cdot & \cdot & \cdot \\ z_{m 1} & z_{m 2} & \cdot & \cdot & \cdot & z_{m n}\end{array}\right]$

be $m \times n$ PDE solution matrix where $m=11$ and $n=557$

The data matrix in Eqn.(16) is a complex matrix, $f f t(u) \in Z^{m \times n}$, associated with $m \times n$ points of the FFT of PDE solution. The Matlab signal processing tool shows that the sampled PDE solution is a complex array consisting of 11 column index vectors each of 557 complex data points. In this section we 
attempt to express the sampled vector signal $c(t)$ in terms of sampling vector signal $x(t)$ as well as data matrix $f f t(u)$ representing the FFT of PDE solution.

$$
\begin{aligned}
c_{1}(t) & =z_{11} s_{1}(t)+z_{21} s_{2}(t)+\ldots+z_{n 1} s_{n}(t) \\
& =\sum_{i=k=1}^{n} z_{i 1} s_{k}(t) \\
c_{2}(t) & =z_{12} s_{1}(t)+z_{22} s_{2}(t)+\ldots+z_{n 2} s_{n}(t) \\
& =\sum_{i=k=1}^{n} u_{i 2} s_{k}(t)
\end{aligned}
$$

$c_{m}(t)=z_{1 m} s_{1}(t)+z_{2 m} s_{2}(t)+\ldots+z_{n m} s_{n}(t)$

$$
=\sum_{i=k=1}^{n} z_{i m} s_{k}(t)
$$

In vector form $c(t)=s(t) \times f f t(u)$ can be written as:

$$
\left[\begin{array}{c}
c_{1}(t) \\
c_{2}(t) \\
\cdot \\
\cdot \\
c_{m}(t)
\end{array}\right]=\left[\begin{array}{cccccc}
z_{11} & z_{12} & \cdot & \cdot & \cdot & z_{1 n} \\
z_{21} & z_{22} & \cdot & \cdot & \cdot & z_{2 n} \\
\cdot & \cdot & \cdot & \cdot & \cdot & \cdot \\
\cdot & \cdot & \cdot & \cdot & \cdot & \cdot \\
\cdot & \cdot & \cdot & \cdot & \cdot & \cdot \\
z_{m 1} & z_{m 2} & \cdot & \cdot & \cdot & z_{m n}
\end{array}\right] \times\left[\begin{array}{c}
s_{1}(t) \\
s_{2}(t) \\
\cdot \\
\cdot \\
\cdot \\
s_{n}(t)
\end{array}\right] ;
$$

\section{Power of the sampled FFT of PDE solution}

$$
P_{c_{1}}=\left|c_{1}(t)\right|^{2}=\left|\sum_{i=k=1}^{n} z_{i 1} s_{k}(t)\right|^{2}
$$

$$
P_{c_{2}}=\left|c_{2}(t)\right|^{2}=\left|\sum_{i=k=1}^{n} z_{i 2} s_{k}(t)\right|^{2}
$$

$$
P_{c_{m}}=\left|c_{m}(t)\right|^{2}=\left|\sum_{i=k=1}^{n} z_{i m} s_{k}(t)\right|^{2}
$$

Total Power of the sampled PDE solution

$$
\begin{aligned}
P_{c} & =\sqrt{\left(P_{c_{1}}\right)^{2}+\left(P_{c_{2}}\right)^{2}+\ldots+\left(P_{c_{m}}\right)^{2}} \\
& =\sqrt{\sum_{j=1}^{m}\left|c_{j}(t)\right|^{2}} \\
& =\sqrt{\sum_{j=1}^{m}\left(\left|\sum_{i=k=1}^{n} z_{i 1} s_{k}(t)\right|^{2}\right)^{2}}
\end{aligned}
$$

\section{Energy of the sampled FFT of PDE solution}

$$
\begin{aligned}
E_{c_{1}} & =\int_{-\infty}^{+\infty}\left|c_{1}(t)\right|^{2} d t \\
& =\int_{-\infty}^{+\infty}\left|\sum_{i=k=1}^{n} z_{i 1} s_{k}(t)\right|^{2} d t \\
E_{c_{2}} & =\int_{-\infty}^{+\infty}\left|c_{2}(t)\right| d t
\end{aligned}
$$

$$
=\int_{-\infty}^{+\infty}\left|\sum_{i=k=1}^{n} z_{i 2} s_{k}(t)\right|^{2} d t
$$




$$
\begin{aligned}
E_{c_{m}} & =\int_{-\infty}^{+\infty}\left|c_{m}(t)\right| d t \\
& =\int_{-\infty}^{+\infty}\left|\sum_{i=k=1}^{n} z_{i m} s_{k}(t)\right|^{2} d t
\end{aligned}
$$

Total energy of the sampled FFT of PDE solution is given by:

$$
E_{c}=\sqrt{\left(E_{c_{1}}\right)^{2}+\left(E_{c_{2}}\right)^{2}+\ldots+\left(E_{c_{m}}\right)^{2}}
$$

Using Eqn.(17) we obtain:

$$
\begin{aligned}
E_{c} & =\sqrt{\sum_{j=1}^{m} \int_{-\infty}^{+\infty}\left|c_{m}(t)\right|^{2} d t} \\
& =\sqrt{\sum_{j=1}^{m}\left(\int_{-\infty}^{+\infty}\left|\sum_{i=k=1}^{n} z_{i 1} s_{k}(t)\right|^{2} d t\right)^{2}}
\end{aligned}
$$

By definition the response of the band pass filter is given by the convolution of the impulse response $w(t)$ of the band pass filter with the sampled vector signal.

$$
c(t)=\left[\begin{array}{lllll}
c_{1}(t) & c_{2}(t) \quad \cdot \quad \cdot c_{n}(t)
\end{array}\right]
$$

Hence we get

$$
w(t)=f(t) * c(t)=\int_{-\infty}^{+\infty} c(t) f(t-\tau) d \tau
$$

Apply the definition to each column, we get:

$$
w_{1}(t)=c_{1}(t) * f(t)
$$

$$
\begin{aligned}
& =\int_{-\infty}^{+\infty} c_{1}(t) f(t-\tau) d \tau \\
& =\int_{-\infty}^{+\infty}\left(\sum_{i=k=1}^{n} z_{i 1} x_{k}(t)\right) f(t-\tau) d \tau
\end{aligned}
$$

$$
\begin{aligned}
& =\sum_{i=k=1}^{n} z_{i 1} \int_{-\infty}^{+\infty} s_{k}(t) f(t-\tau) d \tau \\
& w_{2}(t)=c_{2}(t) * f(t) \\
& =\int_{-\infty}^{+\infty} c_{2}(t) f(t-\tau) d \tau \\
& =\int_{-\infty}^{+\infty}\left(\sum_{i=k=1}^{n} z_{i 2} x_{k}(t)\right) f(t-\tau) d \tau \\
& =\sum_{i=k=1}^{n}\left(z_{i 2} \int_{-\infty}^{+\infty} s_{k}(t) f(t-\tau) d \tau\right) \\
& \begin{aligned}
w_{m}(t) & =c_{m}(t) * f(t) \\
& =\int_{-\infty}^{+\infty} c_{m}(t) f(t-\tau) d \tau \\
& =\int_{-\infty}^{+\infty}\left(\sum_{i=k=1}^{n} z_{i m} s_{k}(t)\right) f(t-\tau) d \tau
\end{aligned} \\
& =\sum_{i=k=1}^{n}\left(z_{i m} \int_{-\infty}^{+\infty} s_{k}(t) f(t-\tau) d \tau\right)
\end{aligned}
$$

\section{Power of the filtered sampled FFT of PDE solution}

$$
P_{w_{1}}=\left|w_{1}(t)\right|^{2}=\left|\sum_{i=k=1}^{n} z_{i 1} \int_{-\infty}^{+\infty} s_{k}(t) f(t-\tau) d \tau\right|^{2}
$$

$$
\begin{aligned}
& P_{w_{2}}=\left|w_{2}(t)\right|^{2}=\left|\sum_{i=k=1}^{n} z_{i 2} \int_{-\infty}^{+\infty} x_{k}(t) f(t-\tau) d \tau\right|^{2} \\
& P_{w_{m}}=\left|w_{m}(t)\right|^{2}=\left|\sum_{i=k=1}^{n} z_{i m} \int_{-\infty}^{+\infty} x_{k}(t) f(t-\tau) d \tau\right|^{2}
\end{aligned}
$$


Total Power of the filtered sampled FFT of PDE solution

$$
\begin{aligned}
P_{w} & =\sqrt{\left(P_{w_{1}}\right)^{2}+\left(P_{w_{2}}\right)^{2}+\ldots+\left(P_{w_{m}}\right)^{2}} \\
& =\sqrt{\sum_{j=1}^{m}\left(\left|w_{m}(t)\right|^{2}\right)^{2}} \\
& =\sqrt{\sum_{j=1}^{m}\left(\left|\sum_{i=k=1}^{n} z_{i 1} \int_{-\infty}^{+\infty} x_{k}(t) f(t-\tau) d \tau\right|^{2}\right)^{2}}
\end{aligned}
$$

Energy of the filtered sampled FFT of PDE solution

$$
\begin{aligned}
& E_{w_{1}}=\int_{-\infty}^{+\infty}\left|w_{1}(t)\right|^{2} d t \\
& =\int_{-\infty}^{+\infty}\left(\left|\sum_{i=k=1}^{n} z_{i 1} \int_{-\infty}^{+\infty} s_{k}(t) f(t-\tau) d \tau\right|^{2}\right) d t
\end{aligned}
$$

$$
\begin{aligned}
E_{w_{2}} & =\int_{-\infty}^{+\infty}\left|w_{2}(t)\right|^{2} d t \\
& =\int_{-\infty}^{+\infty}\left(\left|\sum_{i=k=1}^{n} w_{i 2} \int_{-\infty}^{+\infty} s_{k}(t) f(t-\tau) d \tau\right|^{2}\right) d t \\
E_{w_{m}} & =\int_{-\infty}^{+\infty}\left|w_{m}(t)\right|^{2} d t \\
& =\int_{-\infty}^{+\infty}\left(\left|\sum_{i=k=1}^{n} z_{i m} \int_{-\infty}^{+\infty} s_{k}(t) f(t-\tau) d \tau\right|^{2}\right) d t
\end{aligned}
$$

Using definition and Eqn.(19), the total energy of the filtered sampled FFT of PDE solution is given by:

$$
\begin{aligned}
E_{w} & =\sqrt{\left(E_{w_{1}}\right)^{2}+\left(E_{w_{2}}\right)^{2}+\ldots+\left(E_{w_{m}}\right)^{2}} \\
& =\sqrt{\sum_{j=1}^{m}\left(\int_{-\infty}^{+\infty}\left|w_{m}(t)\right|^{2} d t\right)^{2}} \\
& =\sqrt{\sum_{j=1}^{m}\left(\int_{-\infty}^{+\infty}\left|\sum_{i=k=1}^{n} u_{i 1} \int_{-\infty}^{+\infty} s_{k}(t) f(t-\tau) d \tau\right|^{2} d t\right)^{2}}
\end{aligned}
$$

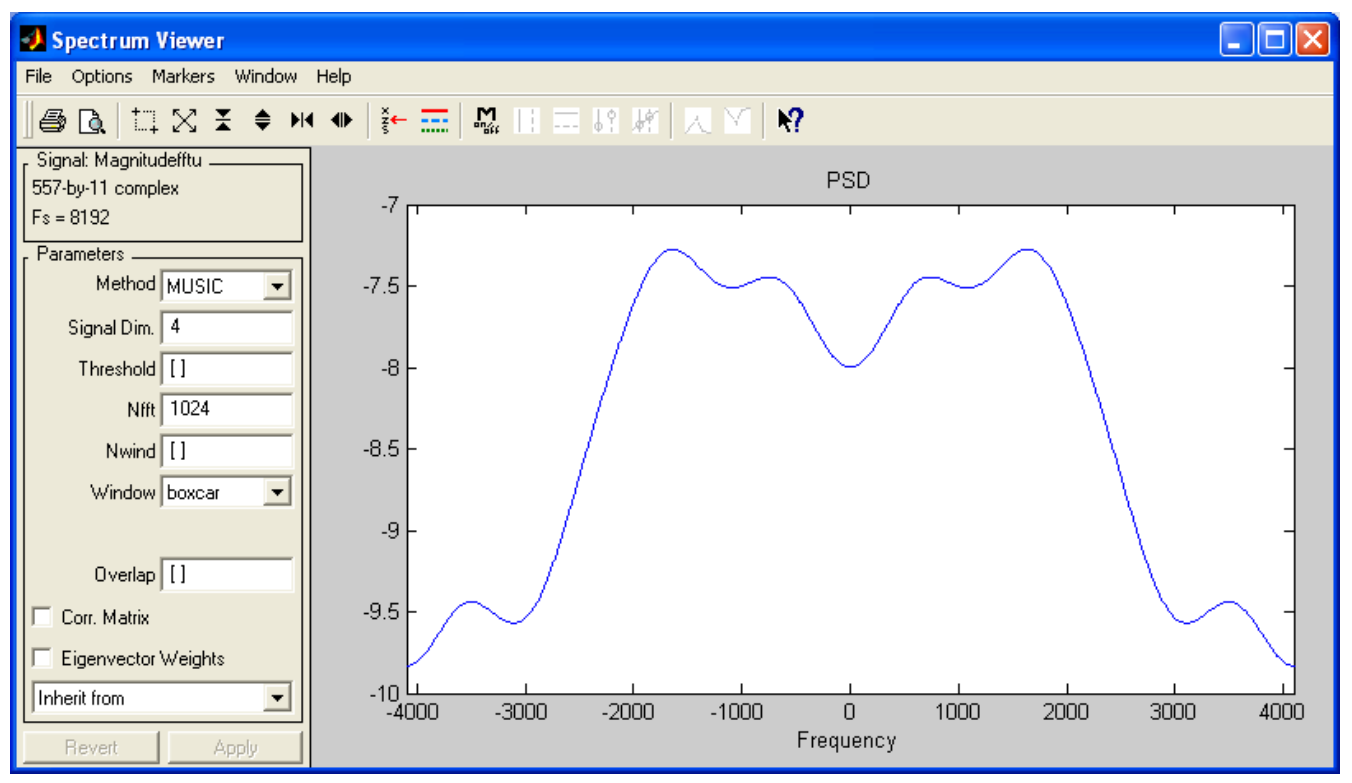

Fig. 17: PSD of fft (u) 


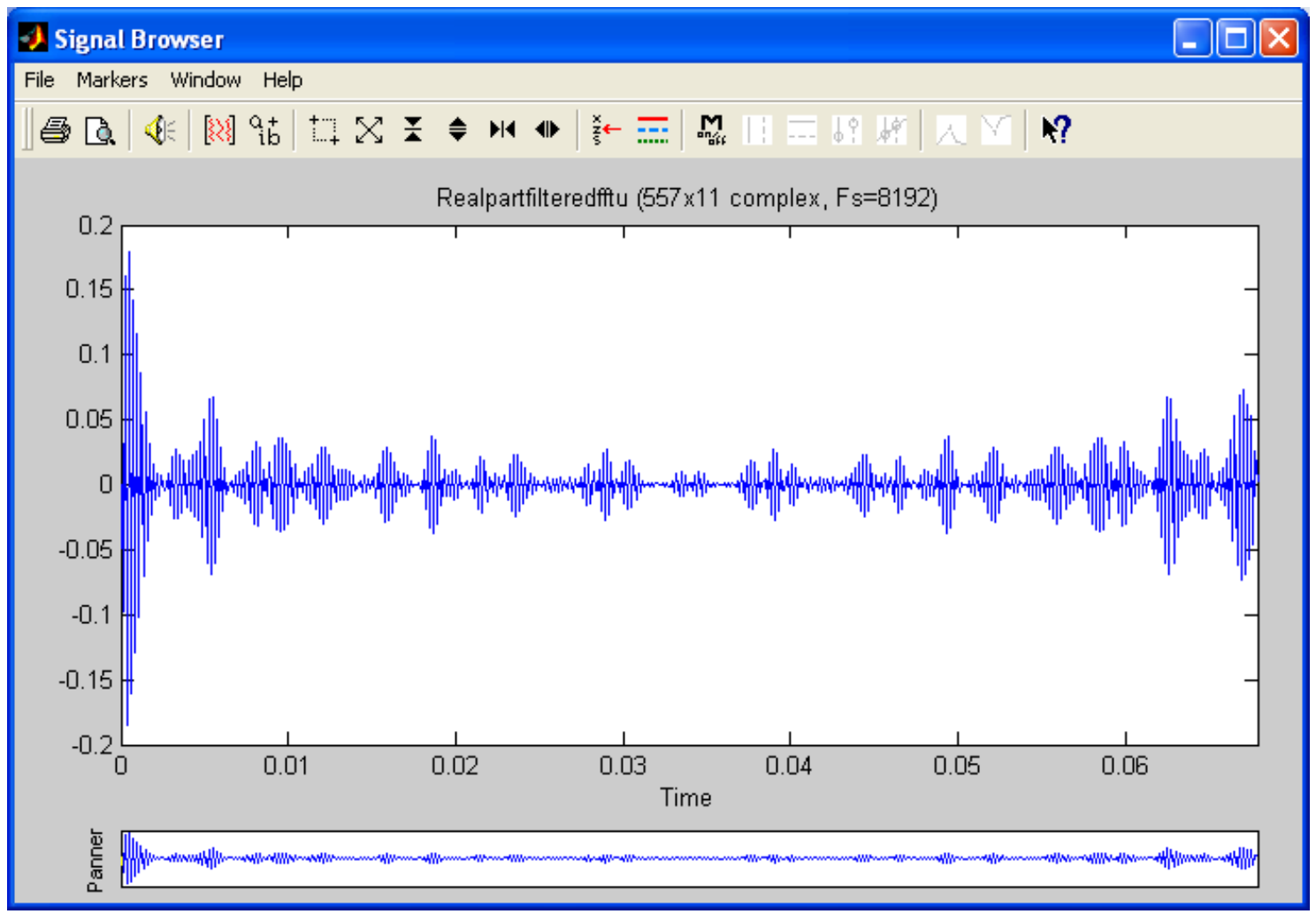

Fig. 18: Real Part of Filtered fft (u)

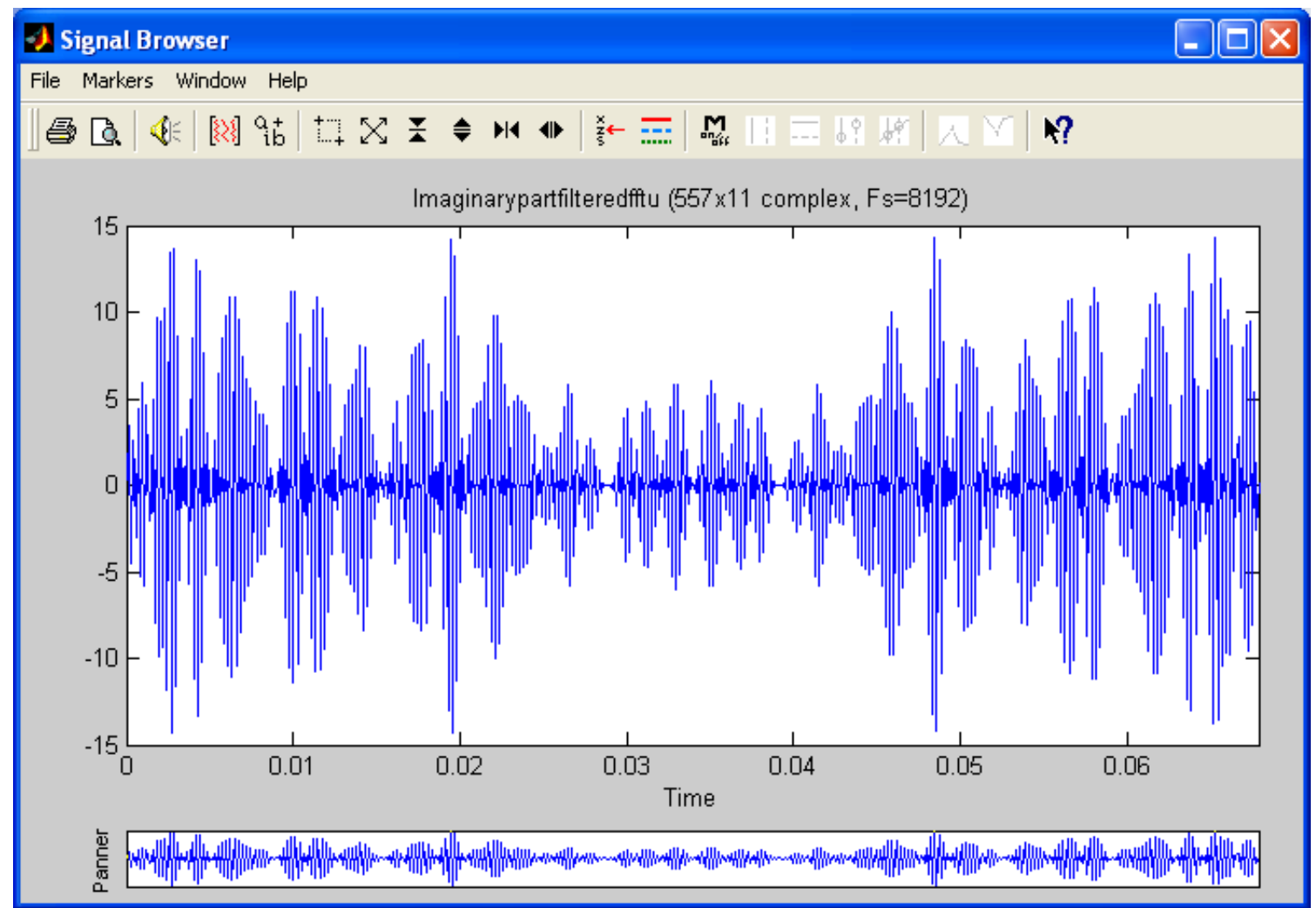

Fig. 19: Imaginary Part of Filtered fft (u) 


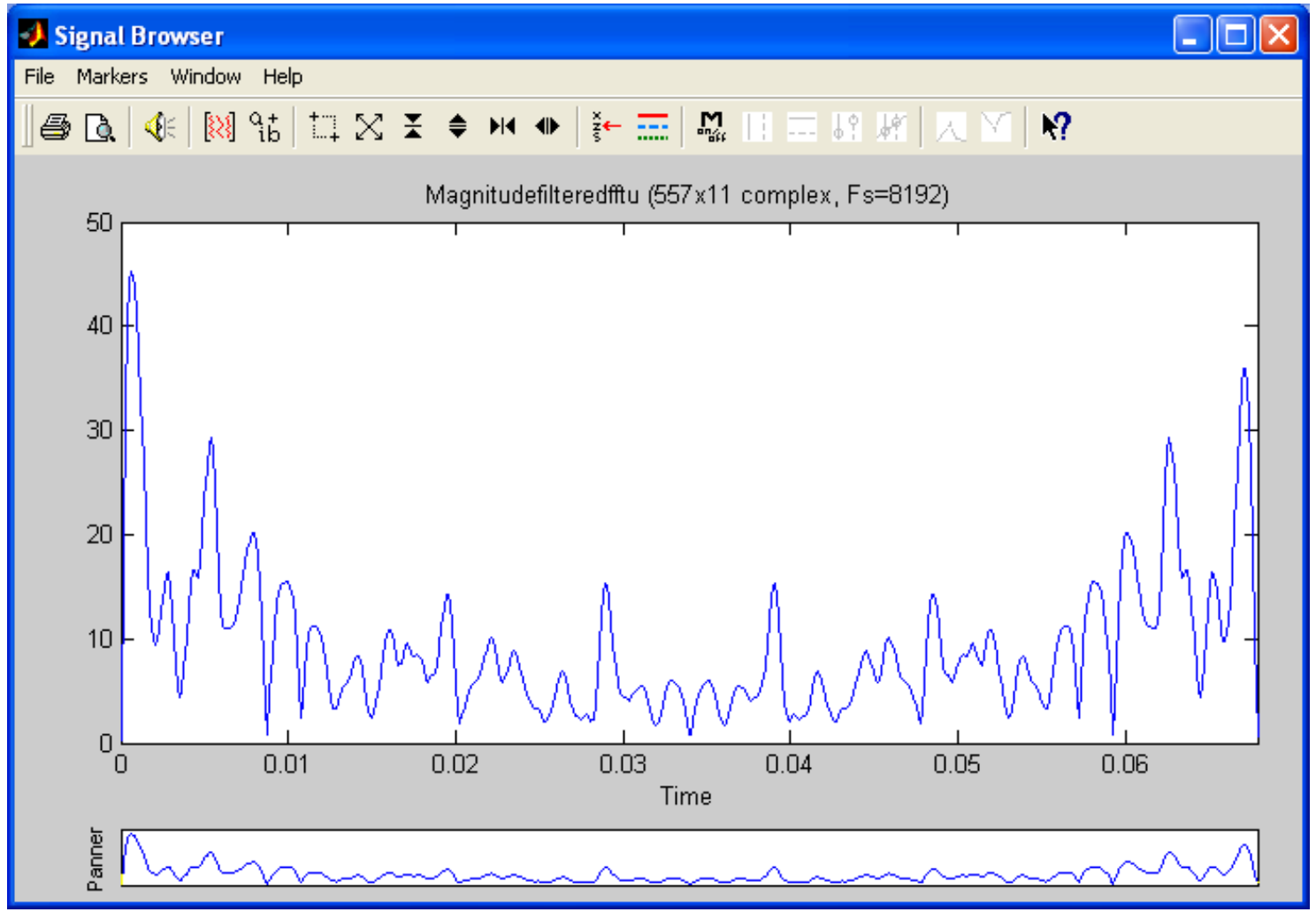

Fig. 20: Magnitude of Filtered fft (u)

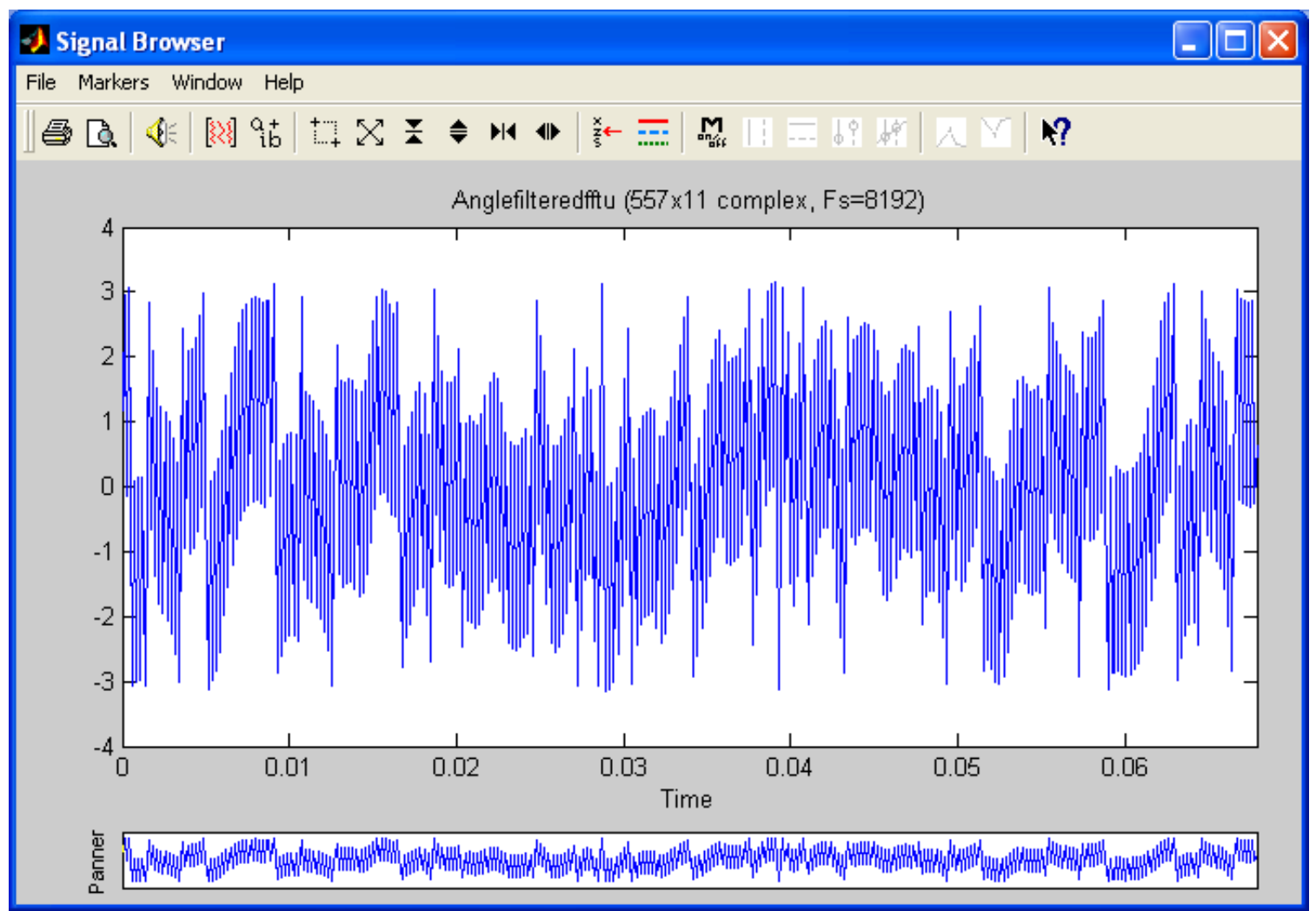

Fig. 21: Phase Angle of Filtered fft (u) 


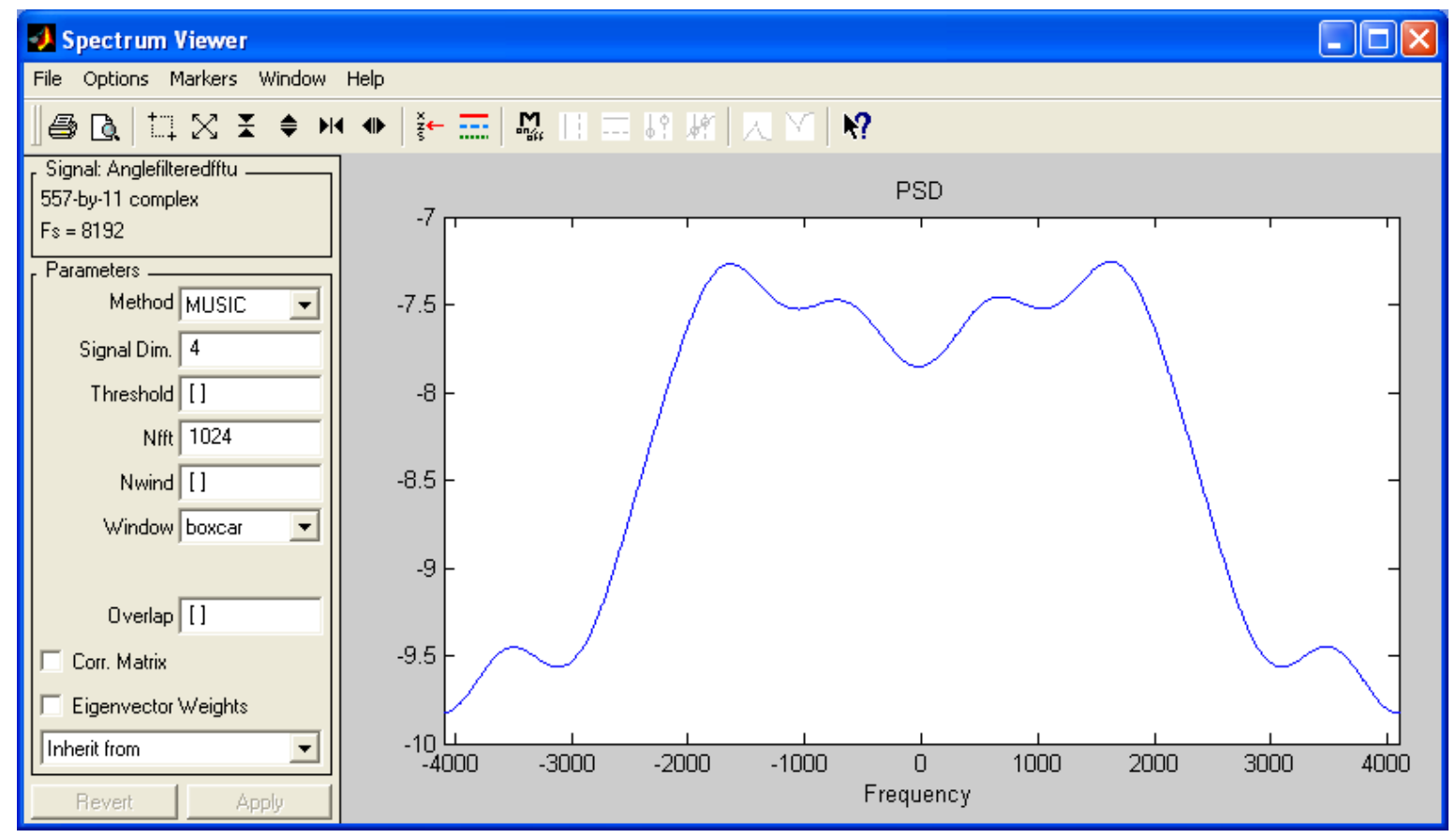

Fig .22: PSD of Filtered fft (u)

\section{Conclusion}

In our research we have proved that the PDE solution generates analogue wavelet transform. We have imported the PDE solution from Matlab workspace to signal processing tool. We have sampled the imported PDE solution and applied the band pass filter to it. The convolution of the sampled PDE solution with the impulse response of the band pass filter has generated analogue wavelet transform. This algorithm computes the analogue wavelet transform either directly of via Fast Fourier Transform. The computation of the FFT of the PDE solution has produced complex wavelet transform. The FFT provides analysis of the PDE solution in frequency domain.

\section{References}

[1] M.Sifuzzaman, M.R.Islam and M. Z.Ali, Application of Wavelet Transform and Its Advantages Compared to Fourier Transform, Journal of Physical Sciences, Vol.13, 2009, 121134

[2] Wells, R.O, Parametrizing Smooth Compactly Supported Wavelet Transform, American Mathematical Society, 338(2): 9019-931, 1993

[3] Strang, G. Wavelets and Dilatation Equations: A Brief Introduction. SIAM Review, 31:614-627, 1989

[4] R99942036, Term Paper, A Tutorial of the Mother Wavelet Transform,
[5] Timothy Herron and John Byrnes, Families of Orthogonal Differential Operators for Signal Processing, 2001

[6] Y. Lyubarskii, Frames in the Bargann Space of Entire Functions, Entire and Subharmonic Functions, 167-180, Adv. Soviet Mth., 11, Amer.Math.Soc, Providence, RI(1992)

[7] K.Seip, Density Theorems for Sampling and Interpolation in the Bargmann-Fork Space I, J.Reine Angew. Math.429, 91-106(1992)

[8] K.Seip, R. Wallstén, Density Theorems for Sampling and Interpolation in the Barg mann-Fork Space II, J.Reine Angew. Math.429, (1992), 107113

[9] K.Seip, Beurling Type Density Theorems in the Unit Disc, Invent. Math. 113, 21-39, 1993

[10] Luis Daniel Abreu, Wavelet Frames, Bergmann Spaces and Fourier Transforms of Laguerre Functions, April 2007

[11] A.J.E.M Janssen, T. Strohmer, Hyperbolic Secants Yield Gabor Frames. Appl. Comput. Harmon. Anal.12, no 2, 259-267 (2002)

[12] A.J.E.M Janssen, Zak Transforms with Few Zeros and the Tie, in 'Advances in Gabor Analysis'(H.G Feichtinger, T.trohmer, eds.), Boston , 2003, ppp.31-70

[13] J. Ramanathan and T. Steger, Incompleteness of Sparse Coherent States. Appl. Comput. Harmon.Anal.2, no 2, 148-153 (1995) 
[14] Dorin Ervin Dutkay and Palle E.T. Jorgensen, Iterated Function Systems, Ruelle Operators, and Invariant Projective Measures, March 2008

[15] E.B. Postinikov and M.V. Lomonosov, Wavelet Transform and Diffusion Equations: Applications to the Processing of the "CASSINI" Space Craft Observations, 2008

[16] Haase M.A , Family of Complex Wavelets for the Characterization of Singularities//Paradigms of Complexity, Ed.M.M Novak.World Scientific, 2000, P.287-288.

[17] E.B. Postnikov, Time-Frequency Analysis of NonStationary Signals Using the Continuous Wavelet Transform Based on the Solving of PDE, XVIII Session of the Russian Acoustical Society, 2006

[18] M.Meléndez-Rodríguez, J. Silva-Martínez and R. Spencer, Efficient Circuit Implementation of Morlet Wavelets, Journal of Applied Research and Technology, Vol.1no1 April 2005.

[19] Stefan Goedecker, Wavelets and their Application for the Solution of Partial Differential Equations in Physics, Max-Planck Institute for Solid State Research, Stuttgart, Germany, May 20, 2009

[20] Amritasu Sinha and Jean Bosco Mugiraneza, Principles of Engineering Analysis, Alpha Science Int'l Ltd, Oxford, UK, 2012, ISBN: 9781842657010

\section{Bibliographies}

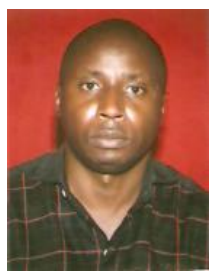

Mr. Jean-B osco Mugiraneza received the BSc in Electromechanical Engineering in 2003 from Kigali Institute of Science and Technology and the M.E. in Electrical Engineering in 2006 from City University of New York. He is actually Member of International Association of Engineers, Member of International Association of Computer Science and Information Technology, Member of Computer Science Teachers Association, Member of International Association of Science and Technology for Development as well as Member of International Society for Engineering Education. He is the co-author of the books on Principles of Engineering Analysis, ISBN: 978-1842657010 and Signals and System Analysis with Matlab and PSpice, ISBN: 978384336681.

Dr. Amritasu Sinha is a Profess or of Mathematics at McMaster University; he was awarded BSc from University of Agra in 1972, MSc from Indian Institute of Technology (Delhi) in 1974, and Ph.D from Indian Institute of Technology (Kanpur) in 1985. He has extensive academic experience in research in Dynamics,
Mathematical Modeling, Complex Analysis and Computational Mathematics. He authored/co-authored numerous books including Applied Differential Equations, ISBN: 9788184870183, Principles of Engineering Analysis, ISBN: 978-1842657010 and Signals and System Analysis with Matlab and PSpice, ISBN: 978384336681.

How to cite this paper: Jean-Bosco Mugiraneza, Amritasu Sinha,"Analogue Wavelet Transform Based the Solution of the Parabolic Equation", International Journal of Information Technology and Computer Science(IJITCS), vol.4, no.12, pp.1-20, 2012. DOI: $10.5815 /$ ijitcs.2012.12.01 